\title{
Persönlichkeitsdissoziation und EMDR-Therapie bei komplexen traumabezogenen Störungen: Anwendungen in Behandlungsphasen 2 und 3
}

\author{
Onno van der Hart \\ Utrecht Universität, Utrecht, Niederlande \\ Mariëtte Groenendijk \\ Top Referent Trauma Center Transit, Mental Health Care Central, Ermelo, Niederlande
}

Anabel Gonzales

Universitätskrankenhaus von A Coruña, Spanien

Dolores Mosquera

INTRA-TP, Instituto para el estudio del Trauma y los Trastornos de la Personalidad A Coruña, Spanien

Roger Solomon

Buffalo Center for Trauma and Loss, Buffalo, New York

\begin{abstract}
Eye Movement Desensitization and Reprocessing (EMDR) - Psychotherapie kann eine bedeutende Rolle in der phasenorientierten Behandlung von komplexen traumabezogenen Störungen spielen. In einem vorangegangenen Artikel wurde die Behandlungsphase $1 \mathrm{im}$ Sinne der Theorie der strukturellen Persönlichkeitsdissoziation und der ihr zugeordneten Aktionspsychologie - Stabilisation, Symptomreduktion und Skills-Training - beschrieben und die Anwendung von EMDR-Verfahren in dieser Phase hervorgehoben. Behandlungsphase 2 beinhaltet im Wesentlichen EMDR-Anwendungen zur Überwindung der Phobie vor traumatischen Erinnerungen und deren anschließende Integration. Behandlungsphase 3 legt den Schwerpunkt auf weitere Persönlichkeitsintegration, was die Überwindung verschiedener das adaptive Funktionieren im Alltag betreffender Phobien mit einschließt. Dieser Artikel hebt Behandlungsansätze hervor, die Therapeuten bei der Integration von EMDR-Protokollen in Phase 2 und 3 einer phasenorientierten Behandlung unterstützen, ohne dabei die integrativen Kapazitäten oder das Toleranzfenster der Patienten ${ }^{1}$ zu überschreiten.
\end{abstract}

Schlüsselwörter: strukturelle Dissoziation; EMDR; traumatische Erinnerungen; Integration; phasenorientierte Behandlung

$\mathbf{W}$ ie in der EMDR-Literatur (z.B., Forgash \& Knipe, 2007; Gelinas, 2003; Hofmann \& Mattheß, 2011; Korn, 2009; Lazrove \& Fine, 1996; Paulsen, 1995, 2007; Shapiro \& Forrest, 1997; Shapiro \& Gelinas, 1999; Twombly, 2000, 2005; Young, 1994) wiederholt betont wurde, müssen EMDR-Behandler ihre therapeutischen Ansätze in eine phasenorientierte Behandlung komplexer traumabezogener

Störungen integrieren. Dies schließt die folgenden, im Diagnostischen und statistischen Manual psychischer Störungen (4. Ausgabe; DSM-IV) aufgeführten dissoziativen Störungen mit ein: Dissoziative Identitätsstörung (DIS) und nicht näher bezeichnete dissoziative Störung (dissociative disorder not otherwise specified, DDNOS, Subtyp 1). Das Standardverfahren (Brown, Scheflin, \& Hammond, 1998;

This article originally appeared as van der Hart, O., Groenendijk, M., Gonzales, A., Mosquera, D., \& Solomon, R. (2014).

Dissociation of the Personality and EMDR Therapy in Complex Trauma-Related Disorders: Applications in Phases 2 and 3 Treatment. Journal of EMDR Practice and Research, 8(1), 33-48. Translated by Marlen Lenzen. 
International Study of Trauma and Dissociation [ISSTD], 2011) wird hierbei meist in drei Phasen gegliedert: (a) Stabilisation, Symptomreduktion, SkillsEntwicklung; (b) die Behandlung der traumatischen Erinnerungen und (c) Persönlichkeits(re)integration und Rehabilitation. Je komplexer die Persönlichkeitsdissoziation, desto weniger werden diese Behandlungsphasen in linearer Abfolge angewendet. Sie sollten über den Behandlungszeitraum eher rekursiv eingesetzt werden; d.h. je nach Bedarf sollte regelmäßig zu einer früheren Phase zurückgekehrt oder auch gelegentlicher ein kurzer Exkurs in die nächste Phase unternommen werden (Courtois, 2010; Korn, 2009; Van der Hart, Nijenhuis, \& Steele, 2006).

Somit sollte der Behandlung traumatischer Erinnerungen - dem Schwerpunkt dieses Artikels - eine Behandlungsphase vorangehen, in der die Grundlage für erfolgreiches und sicheres Prozessieren geschaffen wird. Dies war das Thema unseres vorangegangenen Artikels, in dem die Theorie der strukturellen Persönlichkeitsdissoziation (TSPD) und die ihr zugeordnete Aktionspsychologie als konzeptueller Rahmen verwendet wurden (Van der Hart, Groenendijk, Gonzales, Mosquera, \& Solomon, 2013). Janets in die TSPD integrierte Aktionspsychologie (van der Hart et al., 2006) geht davon aus, dass alle bei Menschen beobachteten psychologischen Fakten im Sinne von behavioralen und mentalen Aktionen ${ }^{2}$ verstanden werden können. Einfache Aktionen sind leicht auszuführen, aber andere, kompliziertere - wie die Integration traumatischer Erinnerungen - erfordern hohe integrative Fähigkeiten(vgl. Van derHartetal., 2006). Als Hauptziele der jeweiligen Behandlungsphasen fordert TSPD die Überwindung spezifischer intro- und extrovertierter Phobien, welche die Persönlichkeitsdissoziation aufrechterhalten (Steele, Van der Hart, \& Nijenhuis, 2005; Van der Hart, Nijenhuis, \& Solomon, 2010; Van der Hart et al., 2006). Die grundlegende, eine Dissoziation aufrechterhaltende Phobie ist die Phobie vor traumatischen Erinnerungen (Janet, 1904), die jedoch bei den meisten Patienten mit komplexen traumabezogenen Störungen nicht als erstes behandelt werden können - daher die Notwendigkeit phasenorientierter Behandlung.

Das Ziel dieses Artikels ist die Erörterung, wie TSPD und die zugeordnete Aktionspsychologie bei der Anwendung von EMDR bei Überlebenden chronischer Traumatisierung während Phase 2 Behandlung traumatischer Erinnerungen (identisch mit Phasen 3-8 des Standard-EMDR-Protokolls) und Phase 3 - Persönlichkeits(re)integration und Rehabilitation - hilfreich sein können.
Wie in früheren Arbeiten beschrieben (vgl. Nijenhuis, Van der Hart, \& Steele, 2002; Steele et al., 2005; Van der Hart et al., 2013; Van der Hart et al., 2010; Van der Hart et al., 2006) wird während eines Traumas die Persönlichkeit des Überlebenden in zwei oder mehr dissoziative Anteile aufgespalten, wobei jeder von ihnen über eine zumindest rudimentäre Ich-Perspektive oder mentale Autonomie verfügt (Nijenhuis \& Van der Hart, 2011). Persönlichkeitsdissoziation spielt sich hauptsächlich zwischen zwei Prototypen dissoziativer Anteile ab, d.h. einem oder mehreren emotionalen Persönlichkeitsanteilen (EP; Myers,; 1940) und einem oder mehreren scheinbar normalen Persönlichkeitsanteilen (apparently normal part of the personality, ANP; Myers, 1940). Die EPs leben in Trauma-Zeit, verharren in traumatischen Reinszenierungen und werden gesteuert durch defensive Aktions-(Sub)Systeme wie Kampf, Flucht und totale Unterwerfung. Die ANPs sind fixiert in der Vermeidung der traumatischen Erinnerungen (und oft auch desinneren Erlebensim Allgemeinen) und werden gesteuert durch Aktionssysteme des Alltagslebens wie Exploration, Fürsorge und Energieregulation. Wird ein ANP gestört durch die traumatische Erfahrung eines EPs, hat die Person dadurch Schwierigkeiten in der Alltags- und Lebensbewältigung. Infolgedessen muss der ANP den EP auf Distanz halten und somit die Persönlichkeitsdissoziation aufrechterhalten. Gemäß der TSPD ist die Trennung der Persönlichkeit in diese Anteile umso stärker, je chronischer die Traumatisierung ist. Die Phobie vor den traumatischen Erinnerungen spielt eine wesentliche (aber nicht die alleinige) Rolle bei der Aufrechterhaltung dieser Teilung; somit besteht ein wesentliches Ziel des EMDR-Behandlungsplans in der Bestimmung von Zeitpunkt und Art der Vorbereitung und Durchführung des Prozessierens oder der Integration - wie es in der TSPD genannt wird - der traumatischen Erinnerungen.

\section{Die Relevanz der Überwindung der Phobie vor traumatischen Erinnerungen für die EMDR-Behandlung}

Die Überwindung der Angst vor traumatischen Erinnerungen ist ein wesentliches Behandlungsziel und beinhaltet das Prozessieren von traumatischen Erinnerungen. Diese Art des Prozessierens wird im Sinne der TSPD als Integration (bestehend aus Synthese und Realisieren ${ }^{3}$ ) traumatischer Erinnerungen verstanden. Das Prozessieren (d.h. Synthese und Realisieren) macht Dissoziation überflüssig (Van der Hart et al., 2010; Van der Hart et al., 2006), obwohl der Patient zeitweise immer 
noch zögern könnte, die verschiedenen dissoziierten Persönlichkeitsanteile loszulassen. Ungeachtet dessen, wie Prozessieren oder Integration der traumatischen Erinnerungen ermöglicht wird, beinhaltet es immer die Fähigkeit des Patienten, integrative mentale Aktionen durchzuführen. Hinsichtlich traumatischer Erinnerungen unterscheidet TSPD zwischen verschiedenen Ebenen des integrativen Prozesses.

Zunächst wird während des Prozessierens (der Integration) der traumatischen Erinnerung letztere synthetisiert; das heißt, sie wird zwischen den dissoziierten Persönlichkeitsanteilen kommuniziert. Auf dieser Ebene stellt das EMDR-Prozessieren (sprich, das EMDR-Standard-Protokoll einschließlich der acht Phasen und drei Stufen, bestehend aus Vergangenheit, Gegenwart und Zukunft; Shapiro, 1989, 2001) einen elementaren integrativen Ansatz dar, in dem Erfahrungen, sprich: Wahrnehmungen, Bewegungen, Gedanken, Gefühle, Affekte, Erinnerungen und eine Ich-Perspektive einerseits gebündelt (verbunden), aber auch differenziert (voneinander unterschieden) werden. Ein Patient könnte zum Beispiel das Problem haben, dass er verunsichert und ängstlich im Umgang mit seinem autoritären Chef reagiert. Die gegenwärtige Situation könnte in Verbindung mit schmerzhaften Kindheitserfahrungen mit einem dominanten Elternteil stehen. Demzufolge können die Erinnerungen an die Vergangenheit und die gegenwärtigen Trigger behandelt werden, so dass der Patient in die Lage versetzt wird, zwischen beiden unterscheiden zu können; ebenso kann eine Zukunftsprojektion installiert werden, die es dem Patienten ermöglicht, sich im Umgang mit seinem Chef geerdet und selbstbestimmt (in control) zu fühlen.

Damit eine vollständige Integration erfolgen kann, muss sich die Synthese jedoch weiterentwickeln zum Realisieren, was manchmal als spontanes Phänomen während einer EMDR-Sitzung auftritt und als Einsicht bezeichnet wird. Realisieren stellt eine höhere Integrationsebene dar (Janet, 1935; Van der Hart et al., 2006). Im Sinne von Janets Aktionspsychologie wird Realisieren definiert als die Entwicklung eines hohen Maßes an persönlicher bewusster Wahrnehmung der Realität, da sie diese anerkennt und sich ihr reflektierend anpasst. Realisieren erfordert viel kognitive und affektive Arbeit, insbesondere zu betrauern, was war, nicht war und niemals wird sein können. Es beinhaltet die Förderung zweier mentaler AktionsTypen: Personifikation und Gegenwarts-Orientierung. Personifikation bedeutet, sich seine persönlichen Erfahrungen und Handlungen zueigen $\mathrm{zu}$ machen (Janet, 1935; Van der Hart et al., 2006); GegenwartsOrientierung, achtsam präsent zu sein, während man sich seines persönlichen Kontextes aus Zukunft und Vergangenheit bewusst bleibt (Janet, 1928; Van der Hart et al., 2006). Um bei dem schon angeführten Beispiel zu bleiben, könnte der Patient hier sagen:

Meine Erfahrungen mit meinem dominanten Vater waren schwierig für mich; sie hatten Einfluss auf mein Selbstgefühl und wie ich mit einigen Situationen umgegangen bin. Aber nun bin ich mir bewusst, dass ich erwachsen bin [sic] und über Optionen und Ressourcen verfüge, die ich als Kind nicht hatte - sogar, wenn ich mich mit meinem Chef auseinandersetzen muss.

\section{EMDR und primäre Persönlichkeitsdissoziation}

Das EMDR Standard-Protokoll kann meist ohne Umschweife bei Patienten eingesetzt werden, die unter einer einfachen PTBS (primären Persönlichkeitsdissoziation) leiden. Da der EP aufgrund seiner rudimentären Ich-Perspektive fast nur aus der traumatischen Erinnerung besteht, beinhaltet das erfolgreiche Reprozessieren der traumatischen Erinnerung automatisch die Verschmelzung von ANP und EP - d.h., dass ihre jeweiligen Erinnerungsnetzwerke vollständig miteinander verschmelzen. Nichtsdestotrotz kann der EP sogar in Fällen einer einfachen posttraumatischen Belastungsstörung (PTBS) durch eine sekundäre Differenzierung gekennzeichnet sein; d.h. über ein etwas erweitertes Repertoire (dys) funktionaler Aktionen, zusätzlich zur Reinszenierung traumatischer Erfahrungen, verfügen. Liegt eine solche sekundäre Differenzierung vor, ist wahrscheinlich mehr Arbeit an der Beziehung zwischen ANP und EP erforderlich, als die bloße Integration der traumatischen Erinnerung.

Als Beispiel sei eine 52-jährigen Frau mit einer primären Persönlichkeitsdissoziation angeführt. Der Therapeut arbeitete mit ihr an ihrer Erinnerung eines fünftägigen Krankenhausaufenthalts im Alter von 5 Jahren, bei dem die Eltern die meiste Zeit nicht anwesend sein konnten, da die Ärzte nur kurze Besuche gestatteten. Während der Sitzung konzentrierte sie sich auf das Bild ihrer selbst nachts im Krankenhaus; sie hatte Angst und das Gefühl: „Ich bin nicht sicher". In der Überzeugung, die Patientin sei nicht wirklich mit den traumatischen Erinnerungen und dem Persönlichkeitsanteil, der diese beinhaltete, in Kontakt, bat sie der Therapeut, in die Augen des Kindes zu sehen. Die Patientin erwiderte: „Das kleine Mädchen wendet sich ab; es hat Angst vor mir." Das kleine Mädchen schien ein EP mit rudimentärer IchPerspektive zu sein. Der Therapeut half der Patientin 
als ANP, mit dem Kind-EP zu kommunizieren, indem er unter anderem Knipes (2007) Liebevolle-AugenMethode (Loving Eyes Procedure) anwendete. Die Patientin war nun als ANP in der Lage, in die Augen des Kind-Anteils zu schauen und daraufhin die Angst, die dieser EP so lang bewahrt hatte, und die zuvor für den ANP unzugänglich gewesen war, zu teilen und somit zu prozessieren. So waren der ANP und der einzelne EP zu einer Integration in der Lage.

\section{EMDR und sekundäre und tertiäre Persönlichkeitsdissoziation}

Bei Patienten mit komplexeren traumabezogenen Störungen und somit einer sekundären oder tertiären Dissoziation sind Modifikationen des EMDRStandard-Protokolls nötig - wie die bereits erwähnten EMDR-Veröffentlichungen zur phasenorientierten Behandlung belegen. Dies würde Modifikationen vieler Verfahrensweisen während der Behandlung in Phase 1 mit einschließen, die auf Stabilisierung, Symptomreduktion und Skills-Training ausgerichtet sind (vgl. Van der Hart et al., 2013). Phase 2, die Behandlung der traumatischen Erinnerung, deren Schwerpunkt auf der Überwindung der Phobie vor traumatischen Erinnerung liegt, beinhaltet ebenfalls eine ganze Reihe modifizierter Verfahren und Interventionen. Hier bedarf die Auflösung der inneren Konflikte des Patienten zwischen Bindung an und Verteidigung gegen den Täter besonderer Aufmerksamkeit, falls die traumatische Erinnerung Missbrauch in der Familie beinhaltet. Solche Konflikte würden eine effektive Reprozessierung stark behindern (siehe folgenden Text).

Vorsichtsmaßnahmen bei der Initiierung von Phase 2, der Behandlung von traumatischen Erinnerungen

Um eine traumatische Erinnerung sicher mit EMDR zu prozessieren (eine angeleitete Synthese im Sinne der TSPD), ist eine graduelle Exposition der dissoziierten Anteile mit der traumatischen Erinnerung erforderlich. Diese abgestufte Exposition muss sich innerhalb der integrativen Kapazität und somit auch der regulativen Toleranz des Patienten abspielen, um maladaptive Reaktionen zu vermeiden (z.B. zusätzliche Dissoziation, Panik, ,Zumachen (Shutting Down) und Vermeidung). Diese regulative Toleranz wurde als Toleranzfenster beschrieben; dieses überschreitend ist der Patient übererregt, darunter ist er untererregt (Ogden, Minton, \& Pain, 2006; Siegel, 1999). Verschiedene EMDR-Fachautoren haben Interventionen vorgeschlagen, um extreme Aktivierung während des EMDR-Prozessierens zu vermeiden (z.B. Fine, 2010; Fine $\&$ Berkowitz, 2001; Lazrove $\&$ Fine, 1996; Luber, 2009, 2010; Twombly, 2000).

Der Therapeut sollte vorsichtig bei der Initiierung dieser Phase sein und mehrere Faktoren bei der Entscheidungsfindung mit dem Patienten in Betracht ziehen. Vorzeitiger Beginn mit dem EMDRProzessieren der traumatischen Erinnerungen kann negative Konsequenzen haben. Ohne eine gründliche Bewertung und ohne sich einen Überblick über die innere, dissoziierte Welt des Patienten verschafft $\mathrm{zu}$ haben und einer darauf folgenden ausreichenden Stabilisierung können etliche Probleme auftreten. Die Patienten können überwältigt werden von Symptomen, intensiven Emotionen oder Schwierigkeiten bei der Alltagsbewältigung; ein Ausagieren kann als Ergebnis einer innerer Kettenreaktion dissoziierter Persönlichkeitsanteile auftreten; oder, etwas weniger offensichtlich: Das Prozessieren kann erfolglos bleiben oder verschiedene Blockaden können es erschweren. Generell kann man sagen: Je niedriger die integrative Kapazität und Energie eines Patienten, desto langsamer sollte diese Behandlungsphase ausgeführt und eine häufige Rückkehr zu Phase 1 (Stabilisierung) mit entsprechenden Interventionen einbezogen werden.

In allen Fällen erfordert Phase 2, die Behandlung traumatischer Erinnerungen, von Patient und Therapeut eine stabile Arbeitsbeziehung (was die Überwindung der Bindungsphobie in Bezug auf den Therapeuten beinhaltet), eine gesunde Motivation und eine realistische Energieinvestition. Für den Patienten bedeutet dies auch konstruktive innere Zusammenarbeit und Empathie zwischen den Persönlichkeitsanteilen, deren Einsatzbereitschaft für EMDR, wie auch die hinreichende Fähigkeit zu ,Ko-Bewusstsein' (some capacity for coconsciousness), Reflektion und Mentalisierung. Mögliche Einwände von Persönlichkeitsanteilen sollten untersucht und beachtet werden. Während sie sich der Persönlichkeitsanteile gewahr sind, die nicht einverstanden sind oder nicht teilnehmen können, sollten sich Therapeuten bewusst sein, dass Entscheidungsfindung bei jedem Schritt des therapeutischen Prozesses auch eine neue Art der Zusammenarbeit innerhalb des internen Systems einleitet, was die Basis für ein effektives und sicheres Trauma-Prozessieren darstellt. Es muss genügend duale Aufmerksamkeit ${ }^{4}$ vorhanden sein, so dass der Patient während des Prozessierens in Kontakt mit der traumatischen Erinnerung bleibt und mit der Gegenwart und dem Therapeuten die Verbindung hält. 
Viele Autoren betrachten einige Situationen als Kontraindikationen für Phase 2, die Behandlung traumatischer Erinnerungen (z.B. Boon, 1997; Gelinas, 2003; Kluft, 1997; Korn, 2009; Lazrove \& Fine, 1996; Steele et al., 2006; Van der Hart et al., 2006; siehe auch EMDR Dissociative Disorders Task Force Guidelines, in Shapiro 1995, 2001). Wesentliche Beispiele hierfür sind andauernder Missbrauch, andauernder Substanzabusus oder anderes selbstschädigendes Verhalten, akute Lebenskrisen, Phasen, in denen zusätzliche Energie und Konzentration im täglichen Leben benötigt wird, Schwangerschaft, hohes Alter, schwere körperliche Krankheit, Psychose, schwere Charakterprobleme, die die Entwicklung eines fokussierten und kollaborativen therapeutischen Behandlungsrahmens verhindern, unkontrolliertes Switching zwischen dissoziierten Persönlichkeitsanteilen und, wenn der Therapeut die einzige Bindungsperson im Leben des Patienten ist. In all diesen Situationen ist meist zunächst weitere Stabilisierungsarbeit erforderlich. Eine Alternative $\mathrm{zu}$ einer langfristigen Stabilisierungsarbeit in diesen Fällen schlagen Gonzales \& Mosquera (2012) in ihrem sogenannten progressiven Ansatz (progressive approach) vor, der im Folgenden beschrieben wird.

\section{Das Prozessieren belastender Empfindungen und Emotionen: Eine Brücke zu Phase 2, der Behandlung traumatischer Erinnerungen}

Der progressive Ansatz (Gonzales \& Mosquera, 2012) ist ein graduelles Prozessieren von Erfahrungen, die mit der traumatischen Erinnerung verbunden sind, wie etwa dissoziative Phobien oder belastende Gefühle und Empfindungen, anstatt die traumatische Erinnerung selbst zu fokussieren (wie im StandardProtokoll). Anders gesagt liegt hier der Schwerpunkt darauf, den Stress abzubauen, der durch die äußeren "Schichten" der traumatischen Erinnerung verursacht wird. So gesehen besteht in der phasenorientierten Behandlung keine klare Trennung zwischen Phase 1 (Stabilisierung) und Phase 2 (Behandlung der traumatischen Erinnerungen), sondern eher ein kontinuierlicher und gemeinsamer Prozess therapeutischer Entscheidungsfindung. Während der Stabilisierungsphase werden weitere kleine Fragmente traumabezogener Aktionen sukzessive entsprechend der integrativen Kapazitäten (Fähigkeit zur Selbstberuhigung, interne und externe Stabilität) des Patienten bearbeitet. Das Prozessieren solcher Aktionen, wie mit dem Trauma verbundener Gefühle und Empfindungen, während die traumatische Erinnerung selbst vermieden wird, könnte als Phase 1
(Stabilisierung) -Intervention betrachtet werden, die den Patienten auf Phase 2 (Phasen 3-8 von EMDR) der Traumaarbeit vorbereitet. In gewisser Hinsicht jedoch kann es auch als "Trauma-Arbeit" verstanden werden, da Therapeut und Patient mit der ,äußeren Schicht" der traumatischen Erinnerung arbeiten. Diese Interventionen haben etwas Ähnlichkeit mit anderen Vorschlägen, die in der Literatur zu finden sind; wie z.B. Knipes (2010) Protokolle zur Arbeit mit Abwehrreaktionen. Im Sinne der TSPD beinhaltet dies auch die Überwindung der Phobie vor der traumatischen Erinnerung.

Die Fingerspitzen-Strategie. Die einem EP zugeordneten, belastenden Gefühle und Empfindungen sind oft mehr oder weniger direkt mit traumatischen Erinnerungen verbunden. Hier kann die Fingerspitzen-Strategie (Tip of The Finger Strategy, TFS; Gonzales \& Mosquera, 2012) hilfreich sein. Das Ziel der TFS ist es lediglich, bestimmte Belastungen eines EPs herunterzufahren, die die Fähigkeit einer besseren Gegenwartsorientierung dieses Persönlichkeitsanteils blockieren, um mit anderen Anteilen zusammenzuarbeiten und seine reflexiven Fähigkeiten auszubauen. Mithilfe der Hand-Metapher, wobei die Handfläche für die traumatische Erinnerung steht, bearbeitet TFS nur einen kleinen Teil einer peripheren Empfindung, eines Gefühls, oder einer irrationalen Überzeugung, die durch die „Fingerspitze" repräsentiert werden kann. Das folgende Fallbeispiel illustriert die Anwendung von TFS, wobei der Patient aufgefordert wird, sich lediglich auf die „Fingerspitzen-Erfahrungen“ zu konzentrieren, während bilaterale Stimulation (BLS) durchgeführt wird.

Brian ist ein 41-jähriger Mann mit schwerem aggressivem Verhalten, das er mit einer feindlichen Stimme in Verbindung bringt. Der Therapeut half ihm, als ANP einen Dialog mit diesem männlichen EP herzustellen; dieser gab an, dass er (der EP) sich stärker fühle, wenn er den ANP dazu dränge, andere Leute zu schlagen. Der EP verachtete den ANP wegen seiner Schwäche: „Er ist nicht mehr als ein Wurm." Nach intensiven Verhandlungen willigte der EP ein, einen Vertrag des „Nicht-Verletzens“ bis zur nächsten Sitzung zu unterzeichnen. Als der Patient wiederkam, hatte er eine andere Person schwer verprügelt. Der EP sagte, dass er den Vertrag hatte erfüllen wollen, aber dass sein innerer Druck so extrem gewesen sei, dass er explodiert sei. Der EP stimmte zu, „einen Teil dieses Drucks“ zu prozessieren, um wieder mehr Kontrolle zu erlangen. Der ANP willigte auch ein, den EP in der Sitzung arbeiten zu lassen. 
TFS wurde als Experiment vorgeschlagen damit der EP überprüfen konnte, ob sie für ihn hilfreich wäre. Der Therapeut erinnerte den EP daran, ggfs. das Stopp-Signal zu nutzen.

Der Therapeut forderte den EP dann dazu auf, sich auf „den Teil deiner Empfindungen, wo du Erleichterung brauchst" zu konzentrieren, und den ANP und den Therapeuten wissen zu lassen, wenn er es bemerkte. Als der Patient ein Signal gab, um seine Bereitschaft zum Start zu zeigen, setzte der Therapeut ein kurzes Set BLS ein, in der Annahme, dass BLS das affektive Arousal senken würde. Dann fragte der Therapeut den EP, wie er sich fühle und der EP antwortete, dass der Druck nachgelassen habe. Zwei Sets wurden durchgeführt, bei denen keine signifikanten Assoziationen oder Einsichten aufkamen. Nach dieser Sitzung verbesserte sich die interne Kommunikation zwischen ANP und EP und die aggressiven Ausbrüche hörten auf. Die belastenden Empfindungen des EPs standen wahrscheinlich in Verbindung zu einer traumatischen Erinnerung, aber der Therapeut ging dem nicht weiter nach. Das Ziel bestand nur darin, einen kleinen Teil des Drucks zu reduzieren. Dennoch wäre es in anderen Fällen ratsam, zunächst den Druck und seinen Ursprung genauer $\mathrm{zu}$ bestimmen: Es könnte z.B. der Einfluss eines täterimitierenden Persönlichkeitsanteils sein, der besonderer Beachtung bedarf.

Wie bei allen anderen Interventionen ist es wichtig, dass sowohl der EP als auch das gesamte System der dissoziierten Anteile dieser Arbeit zustimmen. Die Anwendung von BLS wird in Sets durchgeführt, die kürzer und langsamer als üblich sind, wobei die Wirkung jedes Sets bewertet werden sollte. Bei abnehmender Intensität oder wenn der Patient damit beschäftigt ist, ,adaptiv zu assoziieren', können weitere Sets eingesetzt werden. Bei steigender emotionaler Intensität sollten Selbstberuhigungs-Strategien durchgeführt werden. TFS kann starke Wirkungen erzielen, sogar mit nur einem Set BLS. Somit sollte der erste Einsatz sehr behutsam sein und der Therapeut daran denken, dass eher mehr als weniger der sicherste Weg ist.

\section{Phase 2, Behandlung traumatischer} Erinnerungen: Auflösung der unsicheren Bindung zum Täter

Wenn traumatische Erinnerungen an Missbrauch in der Familie reaktiviert und zum Behandlungsgegenstand werden, verschärft sich der innere Konflikt des Patienten zwischen Bindung zu und Verteidigung gegen den Täter (Steele, Van der Hart \& Nijenhuis,
2001, 2005; Van der Hart et al., 2006). Deshalb sollte der Therapeut den Patienten nicht gegen den Täter positionieren. EPs, die im „Bindungsschrei“ (,attachment cry") gefangen sind (ein BindungsaktivierungsSubsystem, das darauf ausgerichtet ist, Bindung nach einer Trennung von einer Bindungsfigur wiederherzustellen; eine Erfahrung, die der Patient als Kind gemacht haben kann), können ein Verhalten zeigen, das sich nicht per se im Schrei, aber im Festklammern, in maladaptiver Abhängigkeit und Unterwürfigkeit gegenüber dem Täter äußern kann. Gleichzeitig können andere Persönlichkeitsanteile starke Gefühle wie Hass, Wut, Scham oder Schrecken gegenüber dem Täter und anderen empfinden. Einige Patienten können gegenwärtig als ANP mit ihren Familien verstrickt sein; unfähig, sich in gesunder Weise abzugrenzen. Während der Therapeut Gegenwartsorientierung (presentification) fördert, inklusive der Zeitorientierung der dissoziierten Persönlichkeitsanteile, muss er einfühlsam alle widerstreitenden Gefühle und Überzeugungen untersuchen, die mit dem/den Täter/n verbunden sind, ohne Partei zu ergreifen und dabei nicht zu vergessen, dass ein Persönlichkeitsanteil des Patienten mit einer Perspektive auf den Täter identifiziert sein kann (z.B. „Ich hasse meinen Vater für das, was er mir angetan hat!"), wohingegen ein anderer Persönlichkeitsanteil eine ganz andere Ansicht unterstützt („Ich liebe meinen Vater! Er war zuhause der einzige, der sich um mich gekümmert und mir etwas Wärme gegeben hat!"). Der Therapeut sollte diesem Persönlichkeitsanteil empathisch zu der Einsicht verhelfen, dass das Ziel des Prozessierens (der Integration) traumatischer Erinnerungen von Missbrauch nicht ist, den Vater als absolut schlecht zu verdammen, sondern vielmehr, Persönlichkeitsanteilen dabei zu helfen, sich von einem Leben in der Trauma-Zeit zu befreien.

Des Weiteren benötigt der Patient Hilfe dabei, eine integrative Sichtweise bzgl. Persönlichkeitsanteilen zu entwickeln, die an missbräuchliche Betreuungspersonen gebunden und solchen, die diesen gegenüber defensiv fixiert sind. Zur Zeit der Traumatisierung hatten beide Reaktionsmuster Überlebenswert. BLS kann eine solche integrativen Haltung zwischen den Persönlichkeitsanteilen und somit eine größere gegenseitige Akzeptanz befördern. Ist sich der Patient der gegensätzlichen Sichtweisen bewusst, kann dies durchgeführt werden, indem der Patient gebeten wird, sich gleichzeitig auf die Sichtweisen beider Anteile zu fokussieren; dabei werden langsame, kurze BLS-Sets eingebracht.

Der Therapeut muss den Patienten darin anleiten, Grenzen gegenüber missbräuchlichen Menschen 
$\mathrm{zu}$ setzen, die trotz dieser widersprüchlichen Gefühle sowohl emotionale als auch körperliche Sicherheit gewährleisten; dies sollte begleitet werden von Psychoedukation in Bezug auf gesunde Grenzsetzungen (Boon, Steele \& Van der Hart, 2011). Wird der Patient immer noch missbraucht, muss der Therapeut ihn zunächst darin unterstützen, reale Sicherheit herzustellen.

Phase 2, Behandlung traumatischer Erinnerungen: Phasen und Variationen

In Fällen sekundärer und tertiärer Dissoziation kann die Anwendung der EMDR-Therapie zum Prozessieren traumatischer Erinnerungen von Patient zu Patient erheblich variieren, da dissoziative Personen eine ziemlich heterogene Gruppe darstellen. Eine bestimmte EMDR-Adaption kann für einen Patienten gut funktionieren, aber ineffektiv oder sogar dysregulativ für einen anderen sein. Demzufolge müssen Therapeuten flexibel in ihren Behandlungsansätzen traumatischer Erinnerungen sein und ein Arsenal an Werkzeugen und Techniken zur Hand haben und immer behutsam mit Schwierigkeiten in der therapeutischen Beziehung umgehen. Therapeuten müssen mit jedem einzelnen Patienten dahingehend zusammenarbeiten, was am effektivsten und tolerabel in einem stabilen Behandlungsrahmen sein könnte.

\section{Phasen des Prozessierens (der Integration) traumatischer Erinnerungen}

Verantwortungsvolle Behandlung traumatischer Erinnerungen beinhaltet die Aufteilung dieser Hauptaufgabe in eine Reihe mehr oder weniger unterscheidbarer Phasen: Eine eingehende Vorbereitung auf das Verfahren; EMDR-Prozessieren im Sinne einer angeleiteten Synthese (guided synthesis); und EMDR-Prozessieren im Sinne einer angeleiteten Bewusstwerdung, die sich allerdings spontan während des Prozessierens entwickeln kann.

\section{Phase 1: Vorbereitung}

Sorgfältige Vorbereitung des EMDR-Prozessierens im Sinne einer angeleiteten Synthese maximiert die Wahrscheinlichkeit, dass die Arbeit sich innerhalb der Integrationsfähigkeit des Patienten entwickelt und minimiert das Risiko unkontrollierter (defensiver) Einmischung dissoziierter Persönlichkeitsanteile während der Sitzung. Therapeut und Patient haben das Ziel, heftige Emotionen, die per definitionem in- tolerabel sind und selbstschädigendes Verhalten zur Folge haben, zu verhindern. Somit ist der Therapeut gut beraten, mit dem Patienten die möglichen Risiken während dieser intensiven Behandlungsphase zu erörtern. Erörtert werden sollten die Auswirkungen auf den Alltag, das Risiko des Acting Out, Konsequenzen für soziale Beziehungen und das Risiko der Trauma-Reinszenierung. Vor dem Beginn der EMDR-Sitzungen muss der Therapeut mit dem Patienten einen Plan ausarbeiten, wie er danach zurechtkommen kann; dies beinhaltet Erholung und Sicherheit, soziale Unterstützung, angemessene Selbstfürsorge und Selbstberuhigung. Eingeplante verlängerte Sitzungen können hilfreich sein; nicht, um die Intensität oder die Dauer der Erfahrungen zu erhöhen, sondern eher, um die traumatischen Erfahrungen langsamer zu dosieren und dem Patienten viel Zeit zu geben, um sich vor Beendigung der Sitzung wieder zu erden und vollständig in der Gegenwart zu orientieren.

Psychoedukation in Bezug auf den Integrationsprozess der traumatischen Erinnerungen durch EMDR ist für den Patienten notwendig, um zu verstehen, wie der Integrationsprozess strukturiert und ausgerichtet sein wird, welche Behandlungsziele zuerst und welche später kommen und warum; anders gesagt: Wie das Prozessieren gesteuert sein wird. Teil dieses EMDR-Behandlungsplans kann die Konstruktion einer Lebenslinie sein, um die traumatischen Erinnerungen zu ordnen. Dies kann hilfreich sein, um Cluster von traumatischen Erfahrungstypen anzulegen und eine Reihenfolge festzulegen, nach der sie behandelt werden (Lombardo, 2012; Morrow, 2008; Shapiro, 2001). Kriterien für Cluster könnten unter anderem sein: Episoden $^{5}$ (episodes), Täter, Bereiche der negativen Kognitionen (NKs) und involvierte Emotionen. Dennoch ist es am wichtigsten, sich dem Patienten in seiner Art und Weise der Kategorisierung anzuschließen. Die Bearbeitungsabfolge hängt auch stark von den Wünschen und dem gegenwärtigen Leiden des Patienten ab (das durch bestimmte Erinnerungen ausgelöst wird). Stellvertretend für jedes Cluster kann eine repräsentative Erinnerung oder eine selbst erstellte Zusammenfassung der pathogenen Kerne zur Behandlung ausgewählt werden. Beobachtende Persönlichkeitsanteile können hierbei helfen, indem sie eine Helikopter-Sichtweise einnehmen.

Verschiedene strukturierende Vorgehensweisen beim Prozessieren traumatischer Erinnerungen. EMDRProzessieren im Sinne einer angeleiteten Synthese 
kann abhängig von der Integrationsfähigkeit oder den Präferenzen des Patienten entweder eher umfassend (encompassing) und zügig, oder langsam und schrittweise durchgeführt werden. Allerdings empfehlen die meisten Autoren eine sehr langsame Art der Behandlung (z.B. Fine \& Berkowitz, 2001; Gelinas, 2003; Gonzales \& Mosquera, 2012; Knipe, 2010; Lazrove \& Fine, 1996; Paulsen, 1995, 2009; Twombly, 2000, 2005) und betonen das Bedürfnis des Patienten, Stabilität, ein Gefühl der Kontrolle und Beherrschung beizubehalten um somit allmählich die Phobie vor traumatischen Erinnerungen $\mathrm{zu}$ überwinden. Ein Grundprinzip ist es, oftmals Phase 2, die Behandlung traumatischer Erinnerungen, und Phase 1, Stabilisierung, zu alternieren.

Die Frage, an welchem Punkt mit dem Prozessieren der traumatischen Erinnerungen begonnen werden sollte, wurde unterschiedlich beantwortet. Eine gute Übung kann sein, mit einer experimentellen EMDR-Sitzung zu starten, in der ein rezenter, nicht $\mathrm{zu}$ intensiver (mild) und isolierter negativer Vorfall behandelt wird, der den Haupt-ANP und andere Persönlichkeitsanteile, die aus der Distanz zusehen, betrifft. Dies ermöglicht es dem Patienten, sich mit der Methode vertraut $\mathrm{zu}$ machen und gibt dem Therapeuten die Gelegenheit, die Fähigkeit und Eigenheiten des Patienten beim Prozessieren, ebenso wie seine Emotionsregulation einzuschätzen.

Eine ähnliche Strategie ist die Behandlung eines aktuelleren, gegenwärtigen Triggers, der die Lebensbewältigung beeinträchtigt. Diese Strategie kann traumatische Erinnerungen triggern, deren Bewältigung die Kapazitäten des Patienten übersteigt. Eine verfrühte Aktualisierung früher Erinnerungen kann vermieden werden, indem sie zuvor identifiziert werden und klargestellt wird, dass diese Erfahrungen „markiert“ für zukünftige Arbeit und nicht jetzt zugänglich gemacht werden. Es muss die Vereinbarung getroffen werden, daß der Patient bei Aktualisierung der Erlebnisse während des Prozessierens dies dem Therapeut mitteilt und daraufhin zusammen Containment-Strategien eingesetzt werden wie z.B, die Erlebnisse in imaginären Tresoren zu verschließen. In jedem Fall sollten ausführliche assoziative Veränderungen vermieden werden; ein Weg, dies zu erreichen, besteht darin, nach ein paar Sets wieder zum Behandlungsfokus zurückzukehren. Delucci (2010) schlägt vor, gegenwärtige Trigger $\mathrm{zu}$ desensibilisieren, bevor eine Fokussierung auf traumatische Erinnerungen an vergangene Ereignisse stattfindet, wonach sie üblicherweise die frühesten traumatischen Erinnerungen behandelt. Hofmann und Mattheß (2011) regen an, nicht mit einer traumatischen Erinnerung an ein kürzliches oder weiter zurückliegendes Ereignis $\mathrm{zu}$ starten, sondern mit zukünftigen belastenden Ereignissen; z.B. einer anstehenden Konfrontation mit dem Chef, anstatt mit dem zugrundeliegenden Konflikt mit dem Vater, der durch den Einsatz einer Affekt-Brücke zugänglich gemacht werden könnte. In jedem Fall ist äußerste Vorsicht geboten, um zu verhindern, dass dieses Vorgehen verfrüht traumatische Erinnerungen triggert.

Ein weiterer Ansatz besteht darin, mit der Synthese der traumatischen Erinnerung $\mathrm{zu}$ beginnen, die derzeit die größte Belastung im täglichen Leben darstellt oder den gegenwärtigen, hauptsächlichen Vorstellungsgrund ausmacht (z.B. Leeds, 2009; Van der Kolk et al., 2007). Es ist ebenso möglich, dass sich Therapeut und Patient für das ErinnerungsCluster entscheiden, das mit den stärksten und schädlichsten negativen Selbst-Überzeugungen oder pathologischen Kernen assoziiert ist: Bei Erfolg könnte dies eine enorme positive Veränderung in Form eines Generalisierungs-Effekts haben; jedoch muß man hier sehr sicher sein, dass diese Herausforderung den Patienten nicht überfordert. Bei manchen Patienten ist es schließlich das Beste, statt Einhaltung einer bestimmten Reihenfolge, jedes Mal die Erinnerung zu behandeln, die am belastendsten für ihn ist, und seinem natürlichen inneren Prozess zu folgen. In solchen Fällen muss ein hochfunktionaler ANP mit hochintegrativen Fähigkeiten die Führung übernehmen. Generell könnten sich Therapeuten bereitwilliger auf die Fähigkeiten ihrer Patienten verlassen, inklusive derer ,innerer Quelle der Weisheit“ (Krakauer, 2001), als auf ihren eigenen festgelegten Behandlungsplan. Nichtsdestotrotz sollten sie auch darum bemüht sein, eine Mindeststruktur und -richtung aufrechtzuerhalten. Ein dynamisches Gleichgewicht zwischen Flexibilität und Struktur, zwischen dem Problembewusstsein und Kapazitäten des Patienten und dem Einfühlungsvermögen (mindsight) des Therapeuten kann das Tempo der Traumaarbeit bestimmen. Ein Beispiel aus der EMDR-Literatur ist Kitchurs „Strategisches Entwicklungs-Modell für EMDR“ („Strategic Developmental Model for EMDR“; Kitchur, 2000; siehe auch Gelinas, 2003), das für die Behandlung komplexer PTBS entwickelt wurde. Basierend auf der Zusammenarbeit mit dem Patienten und auf dessen spezifische Eigenschaften abgestimmt, entwirft sie ein Abfolgemodell und prozessiert Behandlungsgegenstände gemäß einer Entwicklungs-Abfolge. Ihre Idee ist es, dass jüngere Persönlichkeitsanteile aus Sicherheitsgründen nicht aufgefordertwerdensollten, ,, an therapeutischer Arbeit 
teilzunehmen, die eine spätere Entwicklungsphase betrifft" (S. 6). (Dies kann allerdings auch geschehen, indem diese Persönlichkeitsanteile an inneren sicheren Orten verweilen, wenn die $\mathrm{zu}$ prozessierenden Behandlungsgegenstände traumatische Erinnerungen älterer Anteile betreffen.)

Die Untersuchung der Zielerinnerung. Wenn möglich ist es sinnvoll, den Patienten vorzubereiten, indem der generelle Inhalt der traumatischen Erinnerung inklusive ihrem Anfang und Ende kognitiv untersucht wird (dies bewahrt Patienten davor, ,in der Mitte steckenzubleiben"), ebenso bestimmte Aspekte, die als besonders bedrohlich erlebt werden; diese sind bekannt als pathogene Kerne (Van der Hart et al., 2006) oder Hot Spots (Brewin, 2003). Die Auffassung, dass Erinnerungs-Aspekte pathogen sein können, beruht auf der klinischen Beobachtung, dass es zu einem Fortbestand der traumatischen Erinnerungen kommt, wenn man diese während des Prozessierens übersieht. Es sollte eingehend erörtert werden, welche Aspekte oder Dimensionen der traumatischen Erinnerung als erstes behandelt werden sollten und welche danach (in manchen Fällen muss dies sehr detailliert geschehen; siehe in den folgenden Texten unter „Fraktioniertes Prozessieren"). Dies gelingt oft mit denjenigen (beobachtenden) Anteilen am besten, die die Erinnerung aus einer objektiven Dritte-Person-Perspektive beschreiben, ohne ein Wiedererleben zu evozieren.

Anteile, die für eine Teilnahme noch nicht bereit sind, sollten sich vor einer kognitiven Diskussion des Ereignisses an ihre sicheren Orte zurückgezogen haben. Therapeut und Patient sollten nach Erörterung Einverständnis darüber erzielen, welche Lebensbereiche (z.B. Arbeit, Elternschaft) und welche davon betroffene dissoziierte Persönlichkeitsanteile, wenn nötig und möglich, vor der gegenwärtigen Synthese-Erfahrung geschützt werden sollten. Mit anderen Worten sollte auf die Entscheidungsfindung größten Wert gelegt werden, welche dissoziierten Persönlichkeitsanteile während des Prozessierens anwesend und welche sich an ihrem eigenen inneren sicheren Ort befinden sollten. Van der Hart und Boon (1997) präsentierten das Beispiel von Betty, einer 32-jährigen, hochfunktionalen DIS-Patientin, die zwar in Phase 1 (Stabilisierung) gut zurechtkam, aber dann unter nächtlichen Krisen zu leiden begann, in denen ein EP versuchte, sie mit einem Nylon-Strumpf zu erdrosseln. Während andere dissoziierte Persönlichkeitsanteile an ihren sicheren Orten verblieben, konnte ein beobachtender Anteil dem Therapeuten berichten, dass dies Reinszenierungen einer sechsmonatigen Phase im Alter von 15 Jahren waren, während der sie wiederholt von einem Freund sadistisch missbraucht worden war. Dieser Anteil und der Therapeut konnten gemeinsam 10 unterschiedliche, pathogene Kerne bestimmen, die sukzessive behandelt werden sollten, inklusive der antizipatorischen Ängste und dem größten Schmerz. Sie erörterten ebenfalls, welche Persönlichkeitsanteile während des Prozessierens anwesend sein und welche an ihren eigenen sicheren Orten verbleiben sollten. Die erfolgreiche Synthese dieser 10 Kerne dauerte insgesamt 20 Minuten.

Nicht zu untersuchen, welche Persönlichkeitsanteile in welcher Weise in das zu behandelnde Trauma mit einbezogen waren, kann zu schmerzhaften und vermeidbaren Überraschungen führen: Der Patient kann weiterhin in einem übermäßig erregten Zustand verharren, da andere Persönlichkeitsanteile in dieser traumatischen Erfahrung stecken bleiben.

Die Bestimmung der jeweiligen Rollen der Persönlichkeitsanteile. Für einige Patienten mit DIS oder NNBDS ist Phase 1, Stabilisierung, Symptomreduktion und Skills-Training, insoweit ausreichend, als alle dissoziierten Anteile gleichzeitig an der Synthese (dem Prozessieren) teilnehmen können. Bei den meisten $\mathrm{Pa}$ tienten fokussiert sich die Planung - abgesehen vom Inhalt - auf Entscheidungen darüber, welche Persönlichkeitsanteile zuerst am Prozessieren einer bestimmten traumatischen Erinnerung teilnehmen sollten (oder einer Reihe zugehöriger traumatischer Erinnerungen): (a) $\mathrm{EP}(\mathrm{s})$, die Aspekte der traumatischen Erinnerung tragen, sprich: Persönlichkeitsanteile, die während des traumatisierenden Ereignisses aktiv waren und in z.B. Flucht-, Kampf-, Schreckstarreund/oder (totaler) Unterwerfungs-Reaktionen stecken geblieben sind; (b) Persönlichkeitsanteile (ANP und/oder EPs) mit denen die traumatische Erinnerung während des EMDR-Prozessierens kommuniziert werden kann, und (c) Persönlichkeitsanteile, die abhängig von dem Aktions-(Sub)System, das ihre Handlungen steuert, während oder direkt nach der Synthese eine helfende Rolle einnehmen können wie das Zur-Verfügung-Stellen von Mut, Struktur oder Trost. Das Gewähren von Zuwendung und Trost kann beispielsweise am besten von Seiten eines ANPs geschehen, der zumindest vom Fürsorge-Aktions-System gesteuert wird. Ebenso können Entscheidungen darüber getroffen werden, welche Persönlichkeitsanteile nicht teilnehmen sollten und vielleicht an einen sichereren Ort oder in „einen anderen Raum“ gehen können. Wird eine negative Interaktion mit einem Elternteil prozessiert und wollen oder sollten KindAnteile beispielsweise zunächst nicht anwesend sein, 
können sie von einem Fürsorge-Anteil an einen sicheren Ort gebracht werden.

Kurz zusammengefasst ist gewissenhafte und gründliche Vorbereitung der EMDR-Sitzungen bei dissoziativen Patienten essentiell für deren erfolgreiche Durchführung und der Vermeidung von Komplikationen. Obwohl Phase 1, Stabilisierung, insgesamt als Vorbereitung für Phase 2, die Behandlung der traumatischen Erinnerungen, betrachtet werden kann, mag die hier erwähnte spezielle Vorbereitungsarbeit mehrere Sitzungen in Anspruch nehmen.

\section{Phase 2: EMDR-Prozessierung im Sinne einer angeleiteten Synthese}

Dies ist ein modifizierter (modulated) und kontrollierter therapeutischer Ansatz, bei dem das EMDR-Standard-Protokoll (wenn irgend möglich) eingesetzt wird und dem Patienten insgesamt oder einer Auswahl dissoziierter Persönlichkeitsanteile geholfen wird, in der Gegenwart orientiert zu bleiben, während gleichzeitig die traumatische Erinnerung synthetisiert (prozessiert) wird; d.h. in ihren kognitiven, affektiven, sensomotorischen und behavioralen Komponenten. Prozessieren in diesem Sinne ist der angeleitete Versuch einer gemeinschaftlichen und kontrollierten Reaktivierung der traumatischen Erinnerung und der involvierten EP(s). Nicht jedes einzelne Detail der traumatischen Erinnerung muss kommuniziert oder prozessiert werden. Was jedoch letztendlich unbedingt kommuniziert werden sollte sind die pathogenen Kerne, d.h. die bedrohlichsten Aspekte der traumatischen Erinnerung, die der Patient bisher um jeden Preis vermieden hat. Ein oft übersehener pathogener Kern besteht aus dem Erleben von Scham, einer lähmende Emotion, von der die Patienten meist nicht von selbst berichten. Demzufolge sollte der Therapeut den Patienten zu seinen Erfahrungen mit Scham befragen (Kluft, 2013). Während des EMDR-Prozessierens kommunizieren die involvierten EPs ihre jeweiligen Erfahrungen - mentale und behaviorale Aktionen und deren Inhalte - des traumatisierenden Ereignisses sowohl miteinander als auch mit anderen definierten Anteilen. Diese Persönlichkeitsanteile müssen in der Lage sein, gegenwarts- und therapeutorientiert zu bleiben.

Bei der Bestimmung der pathogenen Kerne ist es wichtig, nah an der Erfahrung des Patienten zu bleiben, statt eigene Urteile zu fällen. Die Therapie von Nadia, die unter schwerer emotionaler Vernachlässigung und sexuellem Missbrauch durch ihren Vater litt und wegen komplexer PTBS (sekundäre Persönlichkeitsdissoziation) in Behandlung war, bot hierfür ein Beispiel. Nach einer Phase 1-Stabilisierung, war der Behandlungsgegenstand für die allererste EMDR-Sitzung (eine Probesitzung) die Erinnerung an einen Unfall. Das fokale Bild war sie selbst, wie sie direkt nach einem Verkehrsunfall mit einem schwer verletzten Bein unter einem großen Fahrzeug lag und ein großes Rad auf ihr Bein drückte. Dem ordnete sie ein Subjektives Belastungsempfinden (Subjective Unit of Disturbance [SUD]) von $8 \mathrm{zu}$. Nadias Antwort, was in diesem Bild den SUD verursachte, erstaunte den Therapeuten. Was sie am meisten belastete, war nicht das große Rad, das grade ihr Bein schwer verletzt hatte, sondern vielmehr ihre Mutter, die auf sie zurannte. (Dieses Beispiel verweist auch auf die Bedeutung der Frage nach dem Ausgang des traumatischen Ereignisses.)

Den Patienten im Toleranzfenster halten. Das EMDR-Prozessieren kann auch zeitlich strukturiert werden durch den Einsatz von langsameren und kürzeren BLS-Sets als gewöhnlich (Forgash, 2010; Shapiro, 2001), längeren Gesprächseinheiten und/ oder Erdungstechniken zwischen den BLS-Sets und der wiederholten Rückkehr zur fokalen Erinnerung. Dem Therapeuten sollte dabei klar sein, dass sogar diese kurzen Sets starke Wirkung haben können und er sollte auf den Einsatz verschiedener Interventionen vorbereitet sein, um ggf. dem Patienten dabei zu helfen, innerhalb seines Toleranzfensters oder seiner integrativen Kapazität zu bleiben. Im Falle einer Übererregung kann es hilfreich sein, den Patienten mithilfe von in Phase 1 (Stabilisierung) erlernten Stabilisierungsübungen $\mathrm{zu}$ erden. Gelingt dies dem Patienten, kann der Therapeut erfragen, welcher Persönlichkeitsanteil aktiviert und was das Thema ist. Vielleicht ist Zeitorientierung, MitgefühlsVerstärkung, oder die Stärkung des therapeutischen Rapports (more therapeutic engagement with the therapist) erforderlich. Darüber hinaus kann Persönlichkeitsanteilen vorgeschlagen werden, sich an innere sichere Orte $\mathrm{zu}$ begeben und auf erforderliche Ressourcen zurückzugreifen.

Gerät der Patient in einen Zustand des Hypoarousal, was Teil der fokalen traumatischen Erinnerung sein kann, sollte der Therapeut per direkter Ansprache Hinweise zum Hier und Jetzt geben. Ständige Installation gegenwärtiger Orientierung und Sicherheit (Constant installation of present orientation and safety, CIPOS; Knipe, 2007) ist eine Strategie, mit der der Therapeut dem Patienten fortlaufend helfen kann, mit einem Gefühl von Sicherheit in der 
Gegenwart geerdet zu sein. Sich geerdet und in der Gegenwart sicher zu fühlen wird während Phase 1, Stabilisierung (EMDR Phase 2, Vorbereitung) vermittelt und kann während der Desensibilisierungsphase (EMDR Phase 4) des Prozessierens je nach Bedarf eingesetzt werden. Ebenso kann der Therapeut dem Patienten helfen, zum Behandlungsgegenstand zurückzukehren; vielleicht mit Hilfe einer angemessenen Berührung, um ihn $\mathrm{zu}$ beruhigen, sich auf Körperwahrnehmungen $\mathrm{zu}$ fokussieren, oder indem er einige Hilfs-Anteile fragt, was hilfreich sein könnte, um die Verbindung mit der traumatischen Erinnerung wiederherzustellen. Im Falle eines schweren Hypoarousal (Kollaps), in dem der Patient mit dem Therapeuten völlig unverbunden zu sein scheint, könnte der Therapeut einen Finger berühren (sofern dies zuvor abgestimmt wurde) und die inneren Anteile bitten, ein wenig den Finger anzuheben, wenn der Therapeut fortfahren soll. Die sinnvollste Intervention ist jedoch, solche Zusammenbrüche zu verhindern. Der Patient kann darin geschult werden, subtile Anzeichen von Verbindungsabbruch, Über- und Untererregung zu erkennen, und diese Anzeichen als ,Stop-Signal'-Ersatz einzusetzen, da es stark traumatisierte Patienten überfordern kann, dies dem Therapeuten klar mitzuteilen (Gonzales \& Mosquera, 2012). Kurze, aber respektvolle und kontrollierte Arbeit ist immer besser, als zu viel zu tun, und der Patient sollte immer wieder daran erinnert werden.

Es ist wichtig, Patienten daran zu erinnern, dass sie „nur kommunizieren müssen, was zu wissen, $\mathrm{zu}$ verstehen und $\mathrm{zu}$ heilen notwendig ist." Kurze Erholungspausen sind sinnvoll (in denen man z.B. vorschlägt: "Sie können jegliche Anspannung loslassen in der Gewissheit, dass Sie zu dieser Zeit und an diesem Ort sicher sind"), oder Anregung zur kontrollierten Atemführung, sowie der Rat, sich abzugrenzen, die eigenen Grenzen zu respektieren, schließlich die Aufforderung, dass sie nicken oder zustimmen, wenn sie bereit sind für den nächsten Durchgang. Letzteres ist besonders wichtig, da viele Überlebende chronischen Kindes-Missbrauchs oder -Vernachlässigung nie Respekt vor ihren Grenzen erfahren haben.

Selbst dann, wenn Persönlichkeitsanteile, die am Prozessieren nicht teilnehmen sollten, sich an ihren inneren sicheren Orten befinden und andere traumatische Erinnerungen in einem imaginativen Tresor eingeschlossen sind, besteht bei einigen Patienten immer noch ein hohes Risiko, dass das Prozessieren einer bestimmten Erinnerung unbeabsichtigterweise andere unbearbeitete Erinnerungen aktiviert, die zu den am Prozessieren beteiligten Persönlichkeitsanteilen gehören. In diesen Fällen ist es ausgesprochen hilfreich, dass der Therapeut wiederholt betont, dass zum jetzigen Zeitpunkt nur diese eine bestimmte, ausdrücklich benannte Erinnerung (oder diese Teilerinnerung) integriert wird - und nichts anderes.

Überaktivierte Persönlichkeitsanteile können bei der (Gegenwarts-, M.L.) Zeitorientierung unterstützt werden (z.B., indem der Therapeut sagt: „Weiß dieser Anteil, dass die Bedrohung vorüber ist und nicht jetzt passiert?") (Forgash, 2010; Twombly, 2005, 2010). Ebenso können hypnotische Anregungen zur Zeitverzerrung, wie etwa das Erleben der abgekürzten Dauer des gegenwärtigen Prozessierens (Synthese) im Vergleich zur Echtzeit und das Erleben der Zeitintervalle als prolongiert im Vergleich zur Echtzeit hilfreich sein. Verschiedene Anregungen sowie heilungsförderliche Imaginationsarbeit kann gegen Ende des Prozessierens (Synthese) angeboten werden.

Um es den Patienten zu ermöglichen, die Praxis mit einer hinreichenden Gegenwartsorientierung zu verlassen, ist die Orientierung an Klufts (1993a) Rule of Thirds eine gute Möglichkeit: Hierbei wird die Sitzung mit dem Haupt-ANP begonnen und an aktuellen Problemen gearbeitet; daraufhin wird mit Persönlichkeitsanteilen gearbeitet (z.B. Prozessieren der traumatischen Erinnerung); anschließend wird dem Patienten geholfen, zum Abschluss zu kommen und sich so weit wie nötig zu reorientieren, was bedeutet, daß der ANP, der zur Sitzung kam, wieder die Handlungskontrolle innehat.

Fraktioniertes Prozessieren (angeleitete Synthese). Dies beinhaltet eine Reihe vergleichsweise deutlich abgestufter Techniken, die Kluft $(1990,2013)$ als fraktionierte Abreaktion bezeichnete. Er leistete in dieser Hinsicht Pionierarbeit unter Einbezug verschiedener hypnotischer Interventionen, wobei die Synthese (das Prozessieren) einer traumatischen Erinnerung oder einer Reihe traumatischer Erinnerungen in mehrere kleinere Schritte aufgeteilt wird, was einige oder sogar viele Sitzungen in Anspruch nehmen kann (Fine, 1993; Gonzales \& Mosquera, 2012; Kluft, 1989, 1990a, 1990b, 1997, 2013; Lazrove \& Fine, 1996; Van der Hart et al., 2006; Van der Hart, Steele, Boon \& Brown, 1993). Diese Verfahrensweise ist angezeigt, wenn die integrative Fähigkeit und Angsttoleranz des Patienten begrenzt sind, aber die Integration einer bestimmten traumatischen Erinnerung zwingend geboten scheint (Kluft, 1990a, 2013).

Es gibt unzählige Variationen fraktionierten Prozessierens (im Sinne einer angeleiteten Synthese). 
Zum Beispiel könnte das Prozessieren anfangs als Teil eines sogenannten Bottom-Up-Ansatzes (Odgen et al., 2006) auf die sensomotorischen Aspekte der traumatischen Erfahrung des EPs begrenzt werden, gefolgt von den zugehörigen Emotionen und den NCs. Das Prozessieren kann sogar auf nur jeweils eine sensorische oder emotionale Dimension beschränkt sein, wie z.B. Schmerz, Angst oder Wut. Es kann auch beschränkt sein auf die Erfahrung jeweils eines EPs und als Teil einer umfassenderen traumatischen Erinnerung kommuniziert werden (z.B. zunächst die Erfahrung des Kampf-EPs anstelle eines Flucht-EPs) oder einen bestimmten Zeitabschnitt der traumatischen Erfahrung beinhalten. Bisweilen bietet die peritraumatische, dissoziative Strukturierung einer traumatischen Erinnerung des Patienten sehr gute Ausgangspunkte. Zum Beispiel waren bei einer DISPatientin Teilaspekte des sexuellen Missbrauchs durch ihren Vater als Mädchen mehreren EPs zugeordnet: es gab Hand-Anteile, Brust-Anteile, Mund-Anteile usw. In den Sitzungen wurde diese sequenziell fokussiert, beginnend mit den Hand-Anteilen, während die anderen Anteile an ihren sicheren Orten verblieben.

Bei emotional sehr intensiven Erinnerungen oder Empfindungen (wie z.B.Schmerz) kann der Therapeut die Sitzung durch kürzere BLS-Sets strukturieren (z.B. durch 5 oder 10 Sets). Bei manchen Patienten allerdings sind eher längere Sets hilfreich, um eine intensive Erinnerung zu prozessieren um ihnen damit die Bewältigung der intensivsten Erregungslevels zu ermöglichen. Der Therapeut muß, entsprechend der Reaktionen und der Affekt-Toleranz des Patienten, die Feinabstimmung der BLS beachten. In diesem Sinne entspricht das EMDR-Prozessieren einem gemeinsamer „Tanz" von Patient und Therapeut, bei dem die Anzahl der BLS-Sets, deren Dauer und Tempo durch die Reaktionen des Patienten bestimmt werden. Schließlich kann eine sehr schrittweise und langsame Kommunikation der Affekte (sharing of affect) außerhalb des eigentlichen Prozessierens während der Sitzungen - angeregt werden, z.B. 5\% des gesamten Affekts pro Tag, oder $1-2 \%$ des Schmerzes einer bestimmten traumatischen Erinnerung (Kluft, 1990b).

Kognitives Einweben. Kognitives Einweben soll das Prozessieren erleichtern durch den Einbau adaptiver Assoziationen, wenn der Patient im Prozessieren blockiert oder außerhalb seines Toleranzfensters gerät (z.B. Fine, 2010; Fine \& Berkowitz, 2001; Gelinas, 2003; Shapiro, 2001). Somit stellt es weder ein fraktioniertes Prozessieren noch eine Synthese dar; auch ist es nicht dafür konzipiert, die zu integrierenden Erinnerungen in ihrer Intensität oder Anzahl zu beschränken. Oft ist wiederholtes kognitives Einweben - ausgerichtet auf den Prozess oder den Inhalt - zur Förderung der Einbindung adaptiver Information erforderlich, d.h. der adaptiven mentalen Aktionen des ANPs. Dies ist besonders in Fällen von sog. Looping und Blockade während des Prozessierens von Bedeutung. Für ein effektives Einweben ist das präzise Timing wichtig; z.B. bei zeit- und realitätsbezogener Verwirrung während des Prozessierens der Erfahrung. Beispiele für Einweben in Bezug auf die Zeitorientierung sind: „Können Sie feststellen, welches Jahr wir haben?"; ,Wie alt sind Sie jetzt?"; „Wie lang ist es her, dass dies geschah?“; „Erinnern Sie sich selbst daran, dass dies ,altes Zeugs“ ist"; "Ihre Eltern sind jetzt tot." Abgesehen von der Zeitorientierung ist das Einweben von SelbstfürsorgeKompetenzen hilfreich; beispielsweise: „Bemerken Sie, was dieser Persönlichkeitsanteil benötigt?“ und „Wie können Sie ihm helfen?" Manchmal ist psychoedukatives Einweben nützlich, nicht nur, um die Entwicklung einer adaptiveren Erwachsenen-Perspektive zu erleichtern, sondern auch, um dem Patienten ein stärkeres Erleben der schützenden und unterstützenden Präsenz des Therapeuten zu ermöglichen und letzten Endes Reflexion und eine umfassende Sicht der Dinge zu fördern; zum Beispiel durch Sätze wie: „,Der EP, der all dies aufbewahrte, ist ein Teil von Ihnen,“ „Tatsächlich, das ist Ihnen passiert,“ und: „Jetzt sind Sie erwachsen und sicher."

Sensomotorisches Einweben im Sinne Janets (1919/1925) inspirierte „Triumphhandlungs“Interventionen aus dem Bereich der sensomotorischen Psychotherapie (Odgen et al., 2006), - dies kann in manchen Fällen hilfreich sein. Diese Interventionen helfen Patienten, blockierte körperliche Bewegungen, die als unvollständige Abwehrreaktionen während der traumatischen Erfahrung begannen, zu vollenden. Dies betraf beispielsweise eine Frau mit einem Hypertonus der rechten Armmuskulatur: Sie konnte diese nur entspannen, als sie dieselben Bewegungen, die sie während ihrer Vergewaltigung als Kind gemacht hatte, wiederholte und abschloss (d.h. Strecken des Armes und Faust ballen, um den Täter abzuwehren). Ist das Prozessieren blockiert oder gerät der Patient außerhalb seines Toleranzfensters, kann der Therapeut dem Patienten überdies bei der, inneren Überprüfung der Situation helfen, indem er die ,Meeting-place-Technik` einsetzt um einzuschätzen, ob ein oder mehrere Persönlichkeitsanteile übererregt sind, Einwände haben oder ob ein neuer Anteil hinzugekommen ist.

Zusammenfassend: innerhalb einer kognitiven Konzeptualisierung und bei Durchführung der beschriebenen Vorbereitungsphase können verschiedene 
EMDR-Modifikationen bei komplex-dissoziativen Patienten höchst effektiv sein im Hinblick auf die tatsächliche Synthese (das Prozessieren) der traumatischen Erinnerungen.

Containment-Strategien zwischen den Sitzungen. Im Allgemeinen sollte das Prozessieren in nachfolgenden EMDR-Sitzungen alle unbehandelten ,Reste' einer zuvor behandelten traumatischen Erinnerung beinhalten. Während der Abschluss-Phase des EMDRProzessierens (Phase 7 desEMDR-Standard-Protokolls) werden Vorsichtsmaßnahmen getroffen, damit diese 'Reste' den Patienten in der Zwischenzeit nicht überwältigen. Beispiele für Containment-Strategien sind: das Verwahren dieser Aspekte in einem imaginativen Tresor und das Herstellen eines Einverständnisses zwischen den dissoziierten Persönlichkeitsanteilen, diese Aspekte zwischen den Sitzungen nicht miteinander zu kommunizieren. Der Patient sollte Anerkennung und Lob für die gemeinsame und harte, bislang geleistete Arbeit erhalten. Das Besprechen von tröstenden, beruhigenden und affektregulierenden Maßnahmen zwischen den Sitzungen ist unerlässlich. Nach den EMDR-Sitzungen kann ein kurzer Nachsorge-Kontakt per Telefon oder eine E-Mail (als eine Form von Containment) hilfreich sein. Zusätzlich zu einem Tagebuch oder einem Protokoll, in dem der Patient weitere Erinnerungen, Trigger, Träume und Ähnliches festhält, kann eine Hausaufgabe das Containment erleichtern; wie z.B. herauszufinden, was die Persönlichkeitsanteile, die während der Sitzung so schwer gearbeitet haben, brauchen und Wege zu finden, diese Bedürfnisse zu erfüllen - beispielsweise einen ANP zu bitten, einem Kind-Anteil, der in der Sitzung tiefe Trauer erlebt und mitgeteilt hat, $\mathrm{Zu}$ spruch und Trost zu spenden. Die nächste Sitzung zu Hause vorzubereiten kann ebenfalls sinnvoll sein. ANPs könnten zum Beispiel herausfinden, welche Persönlichkeitsanteile an der nächsten Sitzung teilnehmen müssen und was diese brauchen, um am EMDRProzessieren teilzunehmen. Zusätzlich hat sich die Empfehlung an den Patienten bewährt, ein Gleichgewicht zwischen dem Fokus auf der integrativen Arbeit auf der einen und dem Beruf, täglichen Aufgaben, Erholung und Freizeitgestaltung auf der anderen Seite anzustreben.

\section{Phase 3: EMDR-Prozessieren als angeleitetes} Realisieren (,Begreifen")

Damit die traumatische Erinnerung eine wirklich narrative autobiographische Erinnerung werden kann, muss sie realisiert, ,begriffen' (realized) werden. Dieses ,Begreifen' ist ein häufig auftretendes, spontanes mentales Ereignis, das während des Prozessierens beobachtet wird. Jedoch muss der Therapeut bei komplex dissoziativen Patienten diese oft bei dieser anspruchsvollen Integrationsarbeit unterstützen, damit sie gelingen kann. Somit sollte der Behandler nach erfolgter Synthese die erarbeiteten Realisierungs-Levels beurteilen und gegebenenfalls mit der Behandlung in dieser Richtung fortfahren. Schließlich ist bei den meisten Patienten der Hauptgrund für den Fortbestand einer bestimmten traumatischen Erinnerung die Phobie vor diesem Realisieren. Beispielhaft hierfür steht eine Mutter, die unter traumatischer Trauer litt, und die eineinhalb Jahre nach dem plötzlichen Tod ihres einzigen Sohnes feststellte: „Die Vorstellung, dass er nie wieder da sein wird, lasse ich nicht an mich heran. Sonst wird man verrückt. Sonst würde man tatsächlich nicht weiterleben wollen" (De Volkskrant Magazin, 24. Dezember 2011). Diese Angst vor dem Begreifen könnte ein eigenständiger Fokus für weiteres Prozessieren sein, begleitet von kognitivem Einweben hinsichtlich Gegenwartsorientierung und Personifikation (um dem Wechsel in einen depersonalisierten State vorzubeugen, Anm. d. Übers.).

Wenn der Patient in der Lage ist, in der Arbeit am Trauma ein hohes Funktionsniveau beizubehalten, dann kann er gegenwartsorientiert bleiben, während er kohärent und flexibel über ein traumatisierendes Ereignis berichtet, ohne dieses dabei erneut $\mathrm{zu}$ durchleben oder in einen depersonalisierten State abzugleiten. Realisierungsarbeit verbessert die Fähigkeit des Patienten, sich im Hier und Jetzt zu ändern und anzupassen. Schlussendlich wird der Patient als Gesamtpersönlichkeit verstehen, dass es sich um ein Ereignis in der Vergangenheit handelt und dass die derzeitige Wirklichkeit sich von der Vergangenheit unterscheidet und viel realer ist; zudem, dass das Ereignis Teil seiner Lebensgeschichte ist und für sein Leben gewisse Konsequenzen hatte oder immer noch hat. Weiterhin muß an der Integration des Narrativs innerhalb jedes einzelnen Persönlichkeitsanteils und diese übergreifend gearbeitet werden. Durch wiederholtes EMDR-Prozessieren kann diese Synthese und Realisierung weiter gefördert werden. Immer, wenn eine traumatische Erinnerung fokussiert wird, können andere Persönlichkeitsanteile, die involviert waren, teilnehmen; vorzugsweise in einer vereinbarten Abfolge, wobei vertiefte Erkenntnis- und BedeutungsEbenen entstehen können. In der Notwendigkeit einer kontinuierlichen Behandlung traumatischer Erinnerungen, um alle Assoziations-Kanäle und beteiligten, dissoziierten Anteile zu prozessieren oder zu integrieren (einschließlich der verschiedenen Ebenen von Synthese und Realisieren), liegt in der Regel einer 
der Unterschiede zwischen der Behandlung früher chronischer Traumatisierung und der Behandlung einer einzelnen traumatisierenden Episode.

\section{Behandlungsphase 3: Persönlichkeits(re) integration und Rehabilitation}

Sofern durch die Arbeit in Phase 2, der Behandlung traumatischer Erinnerungen, die Phobie vor traumatischen Erinnerungen weitgehend überwunden wurde, hat der Patient meist eine insgesamt höhere Integrationsfähigkeit erreicht. Nun kann Phase 3, Persönlichkeits(re)integration und Rehabilitation, eingeleitet werden. Das Realisieren des Geschehenen sollte neue Vorstellungen vom eigenen Selbst, von Betreuungspersonen und der Welt beinhalten. Die Hauptziele von Phase 3 sind wie folgt: (a) Die Überwindung der Phobie vor Fusion (d.h. vor Persönlichkeitsintegration, Anm. d. Übers.), die charakteristisch für einige Patienten ist; (b) Trauerarbeit über all die Verluste, die der Patient im Zusammenhang mit der Traumatisierung erlitten hat und noch erleidet; (c) die Überwindung zusätzlicher Phobien, welche die weitere Persönlichkeitsentwicklung verhindern. Ein viertes, in der EMDR-Therapie und aus der AIPPerspektive sehr vertrautes Ziel ist das Erreichen angemessener, adaptiver und ökologischer Lösungen hinsichtlich der bestehenden Symptomatik; dies beinhaltet den Erwerb neuer Skills, Verhaltensweisen und Selbstüberzeugungen; weiterhin die Optimierung der Fähigkeit, im gegenwärtigen und zukünftigen Lebenskontext adaptiv zu reagieren (Shapiro, 2001). In einigen weniger komplexen Fällen könnte dieser Effekt spontan nach dem EMDR-Prozessieren einer Erinnerung eintreten, aber meist muss diesen Zielen besondere Aufmerksamkeit gewidmet werden.

\section{Überwindung der Phobie vor Fusion}

Bei vielen Patienten können sich einige, in die Integration einer bestimmten Erinnerung involvierte, dissoziierte Persönlichkeitsanteile als unmittelbares Ergebnis eines EMDR-Prozessierens im Sinne einer Synthese und Realisierung zusammenschließen, eins werden (Kluft, 1993b, 2013; Twombly, 2000). Andere Persönlichkeitsanteile haben eine umfassendere Lebensgeschichte und ein breiteres Erlebnisspektrum. Bei ihnen kann ein natürlicher Prozess einsetzen, in dessen Verlauf sie zunehmend diese Erfahrungen mitteilen und der schließlich in eine spontane Fusion mündet, ohne die Notwendigkeit gesonderter therapeutischer Interventionen. Andere Persönlichkeitsanteile wiederum können von einem imaginativen
Fusions-Ritual profitieren, wie beispielsweise, dass sie sich gegenseitig umarmen und eins werden (Kluft, 1993b; Van der Hart et al., 2006).

Da sich eine flexiblere und weniger dissoziative Persönlichkeit entwickelt, können sich dissoziierte Persönlichkeitsanteile besser in der (sicheren) Gegenwart orientieren. Der Haupt-ANP erfährt zunehmend andere Anteile als Teil seines Selbst. Reziproke Amnesien der Persönlichkeitsanteile sind rückläufig. Dies gilt ebenso für die Notwendigkeit der EPs, in Abwehr- oder anderen automatisierten Reaktionen auf Trigger fixiert zu bleiben. EPs werden Unterstützung im täglichen Leben bieten, ANPs können besser mit Emotionen umgehen, und ein natürlicher Prozess allmählicher Vereinigung findet statt. Manche Patienten jedoch bzw. umschriebene Persönlichkeitsanteile, sind auf gegenseitige Abschottung und folglich auf Fusions-Phobie geeicht. Zum Beispiel sollten sich Therapeuten dessen bewusst sein, dass ein Grund für das Wiederauftreten der Fusions-Phobie in dieser Phase darin bestehen kann, dass einer oder mehrere dieser Persönlichkeitsanteile traumatische Erinnerungen beinhalten, welche die Patienten als ANP um jeden Preis vermeiden wollen; wie z.B. die höchst schwierige Erkenntnis, dass ihre eigene Mutter sie von Geburt an oder sogar schon vorher abgelehnt hat; oder Fälle, wo der Patient (als EP) seine eigenen Kinder missbrauchte. Ist dies der Fall, ist letztlich eine Rückkehr zu Phase 2, der Behandlung traumatischer Erinnerungen, erforderlich. Einige Patienten aber brauchen Zeit, bevor sie bereit sind, sich diesen existentiellen Herausforderungen zu stellen; bis dahin widmen sie ihre Aufmerksamkeit und Energie der Konsolidierung des bislang Erreichten.

In dem Maße, wie diese Phobie in Phase 3, Persönlichkeitsintegration, weiterhin manifest ist, kann die Klärung und direkte Bearbeitung dieser Angst mit BLS hilfreich sein (indem z.B. zuvor beschriebene Strategien zur Überwindung dissoziativer Phobien zum Einsatz kommen). Man kann den Patienten auch fragen, wie er sich die zukünftigen Ergebnisse seiner Persönlichkeitsintegration vorstellt, dies zum Behandlungsgegenstand von EMDR machen und so die Angst vor der Fusion überwinden (prozessieren). Diese Vorgehensweise ähnelt dem FlashforwardProtokoll (Logie \& De Jongh, 2014). Dennoch muß bei dissoziativen Patienten darauf geachtet werden, dass sie sich nicht gezwungen fühlen, sich diese Zukunft vorzustellen. Fusions-Phobien können überwältigend sein. Vielleicht kann ein kleines Experiment, bei dem der Patient sich vorstellt, dass ein Persönlichkeitsanteil die Fusion erlebt, einem anderen Anteil dabei helfen, fortzufahren. 
Unabhängig davon, welche Wege zur Persönlichkeitsintegration eingeschlagen werden: Die meisten Patienten mit einer vollständig integrierten Persönlichkeit sind im Umgang mit anstrengenden Lebensereignissen belastbarer als Patienten, welche diesen Weg vorzeitig abbrachen (Kluft, 1993b). Dies bestätigt die Auffassung, dass die graduelle Überwindung der Phobie vor der Fusion der Persönlichkeitsanteile ein wesentliches Behandlungsprinzip ist.

\section{Trauerarbeit}

Bei therapeutischen Behandlungsfortschritten können Patienten Momente der Erleichterung und Freude erleben. Man sollte sich jedoch dessen bewusst sein, dass dies unterbrochen sein kann von erneuter Trauer über erlittene Verluste, je mehr den Patienten bewußt wird, was sie so lange vermisst haben. Trauerarbeit ist von grundlegender Bedeutung während sämtlicher Phasen, vor allem jedoch in Phase 3, Persönlichkeits(re)integration und Rehabilitation, wenn die volle Realisierung erlittener Verluste einsetzt (Van der Hart et al., 1993) und Patienten vor der schwierigeren Herausforderung stehen: Akzeptanz und Resignation, (Janet, 1919/1925). Dies kann erleichtert werden durch Thematisierung der Verluste mit Hilfe der Fokussierung des Standard-Protokolls auf Momente, in denen Verluste bewusst wurden (Shapiro, 2001). Dabei müssen Therapeuten ihre Patienten auch unterstützen, indem sie die Trauerarbeit als Teil des Heilungsprozesses betonen. Patienten sind mit ihrer unwiederbringlich verlorenen Kindheit konfrontiert, der existentiellen Einsamkeit und dem Schmerz, der ertragen werden musste und immer noch ertragen werden muss und mit den verlorenen Beziehungen - für manche Patienten mit der Folge der Partner- oder Kinderlosigkeit, oder keine Zeit, Bildung, Job und Geld zu haben; und sofern sie Kinder haben: alles, was sie ihnen nicht geben konnten, und der Tatsache, dass sie stattdessen so viel Energie auf Vermeidung der oder Kampf mit den Folgen der chronischen Traumatisierung verwenden müssen. Solche Trauerarbeit beinhaltet heftige Verlustgefühle, Schmerz, Trauer, Reue, Kummer, Verzweiflung und Panik, die manchmal in existenzielle Krisen bis hin zu Suizidalität münden (Van der Hart et al., 1993).

Manche Patienten machen die Erfahrung von ,interner' Trauer, weil sie die Beziehung zu ihren dissoziierten Anteilen verloren haben, während sie aber immer noch Schwierigkeiten haben, Beziehungen $\mathrm{zu}$ anderen Menschen herzustellen. Für sie bedeutet somit die Persönlichkeitsintegration eine passagere Intensivierung der Einsamkeit.

\section{Die Überwindung der Phobie vor dem normalen Leben}

Eine gesonderte Herausforderung stellen die in dieser Phase offensichtlicheren Phobien vor dem normalen Leben dar. Das Auftreten dieser Phobien kann einige ansonsten nicht nachvollziehbare Krisen bei Patienten erklären, die sich ansonsten gut entwickelt hatten.

Die Überwindung der Phobie vor gesunder Risikobereitschaft und Veränderung. Die Phobie vor gesunder Risikobereitschaft und Veränderung gründet zum einen in (frühen) Lebenserfahrungen, bei denen Risikobereitschaft und Veränderungen katastrophale Folgen nach sich zogen, andererseits in einer geringeren Integrationskompetenz, welche die Anpassung an sich ständig ändernde Umstände äußerst erschwerte oder verhinderte. In der Tat gibt es viele Lebensaspekte, die vermieden wurden, oder für die die adaptiven Skills fehlen, und denen nun begegnet werden muß. Nach dem Prozessieren (Integrieren) vergangener Traumata und gegenwärtiger Trigger kann der Patient befreit sein, um neue Skills und Methoden des Rapports mit der Welt zu erlernen, die er sich während seiner Kindheit nicht aneignen konnte. Ressourcenentwicklung und -Installation (Ressource Development and Installation, RDI; Korn \& Leeds, 2002) und Zukunftsprojektionen können dem Patienten das zur Verfügung stellen, was für den Umgang mit Herausforderungen des Lebens, der Lebensteilhabe und das Entwicklungswachstum erforderlich ist (Gelinas, 2003; Shapiro, 2011). Die Arbeit mit Flashforwards (d.h. innere Bilder verhängnisvoller Szenarien betreffend eine zukünftige Konfrontation mit einem Objekt oder einer Situation) kann den Patienten von den Erfahrungen befreien, die die Phobie vor dem normalen Leben aufrechterhalten (Logie \& De Jongh, 2014).

Die ÜberwindungderPhobievorIntimität. Patienten müssen auch ihre Phobie vor Intimität überwinden, die in traumatischen Kindheitserlebnissen von Verrat durch die Eltern oder anderen Betreuungspersonen begründet ist (Freyd, 1996) und oft von späteren Partnern wiederholt wird. Intimität ist vielgestaltig: emotional, physisch (nicht sexuell) und sexuell. Die Phobie vor Intimität kann sich auf einige oder all diese Formen beziehen (Steele et al., 2005). In gewissem Maße wurde diese Phobie in früheren Behandlungsphasen in der therapeutischen Beziehung angesprochen, die hoffentlich eine sichere Bindungserfahrung vermittelt hat. In Phase 3, der Persönlichkeits(re)integration und Rehabilitation, ist die Überwindung dieser Phobie in Bezug auf andere Personen ein gesondertes Ziel. 
Patienten können immer noch mit Bedrohungsgefühlen in neuen, sicheren Beziehungen kämpfen und sich sicherer in den vertrauten, unsicheren Situationen und Beziehungen fühlen. Man sollte sie zunächst bei der Überwindung der Angst vor emotionaler Intimität unterstützen und sich erst danach der Angst vor physischer und sexueller Intimität zuwenden, da für Letztere die Erstgenannte Voraussetzung ist. Damit emotionale Intimität auf gesunde Art und Weise erlebt werden kann, müssen Patienten lernen, sowohl ihre eigenen Grenzen klar zu setzen, als auch die anderer zu respektieren (Boon et al., 2011). Wirksame Grenzen reduzieren die Angst vor Intimität, da sie ein Gefühl persönlicher Kontrolle vermitteln und für eine Machtbalance innerhalb einer Beziehung sorgen (Steele et al., 2005).

\section{Ressourcenarbeit}

RDI kann eine große Hilfe bei der Entwicklung der notwendigen Skills in allen Fällen von SkillsEntwicklung und persönlichem Wachstum darstellen (Gelinas, 2003; Korn \& Leeds, 2002). Wie bereits erwähnt, können Zukunftsprojektionen effektivere und adaptivere Reaktionen in Bezug auf zukünftige Belastungen ermöglichen (Hofmann \& Mattheß, 2011; Shapiro, 2001).

Im Allgemeinen sehen wir eher spontane Wechsel von Phase 2 nach 3, Persönlichkeits(re)integration und Rehabilitation, und zurück, da Patienten für gewöhnlich den wachsenden Wunsch verspüren, mit ihrem Leben in der Gegenwart „voranzukommen“. Fortschritte sind an der Fähigkeit erkennbar, inwieweit im täglichen Leben mehr integrative Handlungen initiiert, ausgeführt und erfolgreich abgeschlossen werden, was Freude und Erleichterung auslöst. Dies verhilft den Patienten zu einer verbesserten Integrationsfähigkeit und folglich einem anspruchsvolleren Handlungsniveau (Ellenberger, 1970; Janet, 1919/1925; Ogden et al., 2006; Van der Hart et al., 2006). Dies beinhaltet die vollständige Realisierung ihrer eigenen Lebensgeschichte, ihrer gegenwärtigen Lebensumstände und die Vorbereitung auf eine in realistischem Sinne optimistische Zukunft.

\section{Behandlungsabschluss}

Obgleich all dies den Patienten befähigt, ein adaptiveres und erfüllteres Leben $\mathrm{zu}$ führen, ist ein angemessener Behandlungsabschluss eine bedeutende Schwelle, der sorgfältige und nachhaltige Aufmerksamkeit vom Therapeuten und Patienten gewidmet werden muß. Themen, die unter anderem behandelt werden sollten, sind die Überwindung der
Bindungsverlustphobie, die Würdigung der Therapie und des Therapeuten im Leben des Patienten und die Auflösung der therapeutischen Beziehung; es beinhaltet auch die kritische Untersuchung unaufgelöster Gegenübertragungsthemen durch den Therapeuten. Die erfolgreiche Durchführung dieser Therapiephase ist für einen gelungenen Therapieabschluss notwendig (Van der Hart et al., 2006).

\section{Schlussfolgerung}

Für chronisch traumatisierte Patienten und Patienten mit einer komplexen Persönlichkeitsdissoziation stellt die Integration traumatischer Erinnerungen - einschließlich deren vollständiger Realisierung eine große Herausforderung dar, die ihre Integrationsfähigkeit schnell überfordern kann. Aus diesem Grund muss die EMDR-Behandlung traumatischer Erinnerungen - was auf andere Behandlungsansätze ebenso zutrifft - Teil einer sehr viel umfassenderen Therapie sein, die meist als phasenorientierte Behandlung definiert wird. Bei einer solchen Therapie ist die hohe Qualität der therapeutischen Beziehung ausschlaggebend. Dies erfordert eine höchst respektvolle und mitfühlende Haltung des Therapeuten, sowie die Entwicklung einer „Teamarbeit“ von Therapeut und Patient, die schließlich alle dissoziierten Persönlichkeitsanteile des Patienten mit einbezieht. Schwere Traumatisierung beinhaltet extreme $\mathrm{Zu}-$ stände von Verlassen-Sein, Einsamkeit und Hilflosigkeit. Die sichere Präsenz des Therapeuten während der heilsamen Reise ist der wesentliche Wirkfaktor für den Erfolg der Behandlung.

In diesem Artikel lag der Schwerpunkt auf dem Beitrag, den TSPD zu effektiven und sicheren EMDR-Praktiken bei der phasenorientierten Behandlung leisten kann, insbesondere in Phase 2 (der Behandlung traumatischer Erinnerungen) und Phase 3 (Persönlichkeits(re)integration und Rehabilitation) bei Patienten mit komplexen, traumabezogenen Störungen. Das AIP-Modell bleibt maßgeblich als Wegweiser zur Erreichung verschiedener therapeutischer Ziele, wie z.B. die FallKonzeptualisierung, welche die Identifizierung traumatischer Erinnerungen und gegenwärtiger Trigger beinhaltet, und die Erstellung einer positiven Zukunftsprojektion (Shapiro, 2001). Die TSPD und die ihr zugeordnete Aktionspsychologie bieten eine differenziertere kognitive Konzeptualisierung für die jeweiligen Behandlungsziele, die im Wesentlichen in der Überwindung spezifischer Phobien bestehen, die die Persönlichkeitsdissoziation und somit die Existenz der traumatischen Erinnerungen aufrechterhalten. Im 
Rahmen der phasenorientierten Behandlung, angeleitet durch AIP und TSPD, und mit Hilfe sorgfältiger Indikation, Timing und Vorbereitung, kann EMDRTherapie auf flexible, sichere und effektive Weise durchgeführt werden. Nicht nur werden die traumatischen Erinnerungen vollständig integriert, sondern es kommt ebenso zu einer zunehmenden Verschmelzung dissoziierter Persönlichkeitsanteile und zu einer zunehmenden Integration der Gesamtpersönlichkeit. Darüber hinaus werden die Patienten immer kompetenter im Umgang mit alltäglichen Herausforderungen und entwickeln ein positiveres Selbstwertgefühl.

\section{Fußnoten}

1. Anm. d. Übers.: Für einen erleichterten Lesefluss wird im Folgenden in Bezug auf den Patienten nur das Maskulinum verwendet.

2. Anm. d. Übers.: Es gibt im Deutschen keine wirklich treffende Übersetzung für „,mental -“ oder „,behavioral action“, deshalb habe ich den englischen Ausdruck analog übernommen.

3. Anm. d. Übers.: der deutsche Terminus ,Bewußtwerdung trifft den Bedeutungsumfang des englischen realization nur unzureichend, da dieser neben dem kognitiven Anteil auch und vor allem den affektiven impact beinhaltet, der andeutungsweise im deutschen ,Begreifen ' anklingt. Deshalb wird im folgenden ,Realisieren/Realisierung verwendet.

4. Anm. d. Übers.: Unterscheidungsfähigkeit zwischen traumatischer Vergangenheit und sicherer Gegenwart.

5. Anm. d. Übers.: Unklar bleibt, ob hier eine zeitliche oder eine thematische Episode (z.B. Missbrauch durch ein Familienmitglied vom 7.-9. Lebensjahr) gemeint ist.

\section{Literaturhinweise}

Boon, S. (1997). The treatment of traumatic memories in DID: Indications and contraindications. Dissociation, 10, 65-79.

Boon, S., Steele, K., \& Van der Hart, O. (2011). Coping with trauma-related dissociation: Skills training for clients and therapists. New York, NY: Norton.

Brewin, C. R. (2003). Posttraumatic stress disorder: Malady or myth? New Haven, CT: Yale University Press.

Brown, D., Scheflin, A. W., \& Hammond, D. C. (1998). Memory, trauma treatment, and the law. New York, NY: Norton.

Courtois, C. A. (2010). Healing the incest wound: Adult survivors in therapy (2nd ed.). New York, NY: Norton.

Dellucci, H. (2010, June). No matter how slow you go, as long as you don't stop: A six gear mechanics for a safe therapy journey through complex trauma. Paper presented at the 11th EMDRIA Conference, Hamburg, Germany.

Ellenberger, H. F. (1970). The discovery of the unconscious. New York, NY: Basic Books.

Fine, C. G. (1993). A tactical integrationist perspective on the treatment of multiple personality disorder. In $\mathrm{R}$.
P. Kluft \& C. G. Fine (Eds.), Clinical perspectives on multiple personality disorder (pp. 135-154). Washington, DC: American Psychiatric Press.

Fine, C. G. (2010). The wreathing protocol: The imbrication of hypnosis and EMDR in the treatment of dissociative identity disorder, dissociative disorder not otherwise specified, and post-traumatic stress disorder. In M. Luber (Ed.), EMDR, dissociative disorders, and complex post-traumatic stress disorder (pp. 329-348). New York, NY: Springer Publishing.

Fine, C. G., \& Berkowitz, A. S. (2001). The wreathing protocol: The imbrication of hypnosis and EMDR in the treatment of dissociative identity disorder and other dissociative responses. American Journal of Clinical Hypnosis, 43, 275-290.

Forgash, C. (2010). Orienting the ego state system to present reality (OPR). In M. Luber (Ed.), EMDR, dissociative disorders, and complex post-traumatic stress disorder (pp. 225-232). New York, NY: Springer Publishing.

Forgash, C., \& Knipe, J. (2007). Integrating EMDR and ego state treatment for clients with trauma disorders. In C. Forgash \& M. Copeley (Eds.), Healing the heart of trauma and dissociation with EMDR and ego state therapy (pp. 1-59). New York, NY: Springer Publishing.

Freyd, J. J. (1996). Betrayal trauma: The logic of forgetting childhood trauma. Cambridge, MA: Harvard University Press.

Gelinas, D. J. (2003). Integrating EMDR into phase-oriented treatment for trauma. Journal of Trauma \& Dissociation, 4(3), 91-135.

Gonzalez, A., \& Mosquera, D. (2012). EMDR and dissociation: The progressive approach [English edition]. Charleston, SC: Amazon Imprint.

Hofmann, A., \& Mattheß, H. (2011). EMDR bei schweren dissoziativen Störungen. In L. Reddeman, A. Hofmann, \& U. Gast (Eds.), Psychotherapie der dissoziativen Störungen (pp. 135-146). Stuttgart, Germany: Thieme.

International Society for the Study of Trauma and Dissociation (ISSTD). (2011). Guidelines for treating dissociative identity disorder in adults, third revision. Journal of Trauma \& Dissociation, 12(2), 115-187.

Janet, P. (1904). L'amnésie et la dissociation des souvenirs par l'émotion. Journal de Psychologie, 1, 417-453.

Janet, P. (1925). Les médications psychologiques [Psychological healing]. Paris: Félix Alcan. New York, NY: Macmillan. (Original work published 1919)

Janet, P. (1928). L'évolution de la mémoire et de la notion du temps. Paris, France: A. Chahine.

Janet, P. (1935). Réalisation et interprétation. Annales Médico-Psychologiques, 93, 329-366.

Kitchur, M. (2000). The strategic developmental model for EMDR. The EMDRIA Newsletter, Special Edition, 5(5), 4-10.

Kluft, R. P. (1989). Playing for time: Temporizing techniques in the treatment of multiple personality. American Journal of Clinical Hypnosis, 32, 90-98.

Kluft, R. P. (1990a). The fractionated abreaction technique. In C. D. Hammond (Ed.), Handbook of hypnotic suggestions (pp. 527-528). New York, NY: Norton. 
Kluft, R. P. (1990b). The slow leak technique. In C. D. Hammond (Ed.), Handbook of hypnotic suggestions (pp. 529-530). New York, NY: Norton.

Kluft, R. P. (1993a). Basic principles in conducting the psychotherapy of multiple personality disorders. In R. P. Kluft \& C. G. Fine (Eds.), Clinical perspectives on multiple personality disorder (pp. 19-50). Washington, DC: American Psychiatric Press.

Kluft, R. P. (1993b). Clinical approaches to the integration of personalities. In R. P. Kluft \& C. G. Fine (Eds.), Clinical perspectives on multiple personality disorder (pp. 101-133). Washington, DC: American Psychiatric Press.

Kluft, R. P. (1997). On the treatment of traumatic memories of DID clients: Always? Never? Sometimes? Now? Later? Dissociation, 10, 80-90.

Kluft, R. P. (2013). Shelter from the storm: Processing the traumatic memories of DID/DDNOS patients with the fractionated abreaction technique. North Charleston, SC: CreateSpace.

Knipe, J. (2007). Loving eyes: Procedures to therapeutically reverse dissociative processes while preserving emotional safety. In C. Forgash \& M. Copeley (Eds.), Healing the heart of trauma and dissociation with EMDR and ego state therapy (pp. 181-225). New York, NY: Springer Publishing.

Knipe, J. (2010). "Shame is my safe place": Adaptive information processing methods of resolving chronic shamebased depression. In R. Shapiro (Ed.), EMDR solutions II: For depression, eating disorders, performance, and more (pp. 49-89). New York, NY: Norton.

Korn, D. L. (2009). EMDR and the treatment of complex PTSD. Journal of EMDR Practice \& Research, 3, 264-278.

Korn, D. L., \& Leeds, A. M. (2002). Preliminary evidence of efficacy for EMDR resource development and installation in the stabilization phase of treatment of complex posttraumatic stress disorder. Journal of Clinical Psycho$\log y, 58,1465-1487$.

Krakauer, S. Y. (2001). Treating dissociative identity disorder: The power of the collective heart. Philadelphia, PA: Brunner-Routledge.

Lazrove, S., \& Fine, C. G. (1996). The use of EMDR in patients with dissociative identity disorder. Dissociation, 9 , 289-299.

Leeds, A. M. (2009). A guide to the standard EMDR protocols for clinicians, supervisors, and consultants. New York, NY: Springer Publishing.

Logie, R., \& De Jongh, A. (2014). The "Flashforward procedure": Confronting the catastrophe. Journal of EMDR Practice and Research, 8(1), 25-32.

Lombardo, M. (2012). EMDR target timeline. Journal of EMDR Practice and Research, 6(1), 37-46.

Luber, M. (Ed.). (2009). EMDR scripted protocols: Special populations. New York, NY: Springer Publishing.

Luber, M. (Ed.). (2010). EMDR, dissociative disorders, and complex post-traumatic stress disorder. New York, NY: Springer Publishing.

Morrow, R. D. (2008). EMDR target tracking. Journal of EMDR Practice and Research, 2(1), 69-72.
Myers, C. S. (1940). Shell shock in France 1914-1918. Cambridge, MA: Cambridge University Press.

Nijenhuis, E. R., \& Van der Hart, O. (2011). Dissociation in trauma: A new definition and comparison with previous formulations. Journal of Trauma \& Dissociation, 12(4), 416-445.

Nijenhuis, E. R., Van der Hart, O., \& Steele, K. (2002). The emerging psychobiology of trauma-related dissociation and dissociative disorders. In H. D'haenen, J. A. den Boer, \& P. Willner (Eds.), Biological psychiatry (pp. 1079-1098). Chicester, United Kingdom: Wiley.

Ogden, P., Minton, K., \& Pain, C. (2006). Trauma and the body: A sensorimotor approach to psychotherapy. New York, NY: Norton.

Paulsen, S. (1995). Eye movement desensitization and reprocessing: Its cautious use in the dissociative disorders. Dissociation, 8, 32-44.

Paulsen, S. (2007). Treating dissociative identity disorder with EMDR, ego state therapy, and adjunct approaches. In C. Forgash \& M. Copeley (Eds.), Healing the heart of trauma and dissociation with EMDR and ego state therapy (pp. 141-179). New York, NY: Springer Publishing.

Paulsen, S. (2009). Looking through the eyes of trauma \& dissociation: An illustrated guide for EMDR clinicians and patients. Bainbridge Island, WA: Bainbridge Institute for Integrative Psychology.

Shapiro, F. (1989). Efficacy of the eye movement desensitization procedure in the treatment of traumatic memories. Journal of Traumatic Stress, 2, 199-223.

Shapiro, F. (1995). Eye movement desensitization and reprocessing: Basic principles, protocols, and procedures. New York, NY: Guilford Press.

Shapiro, F. (2001). Eye movement desensitization and reprocessing: Basic principles, protocols, and procedures (2nd ed.). New York, NY: Guilford Press.

Shapiro, F., \& Forrest, M. S. (1997). EMDR: The breakthrough therapy for overcoming anxiety, stress, and trauma. New York, NY: Basic Books.

Shapiro, F., \& Gelinas, D. J. (August 1999). Integrating phaseoriented treatment with EMDR: An approved workshop for certification. Tenth Annual Summer Seminar for Mental Health Professionals. Harvard Medical School \& Massachusetts Mental Health Center, N. Falmouth, MA.

Siegel, D. J. (1999). The developing mind: How relationships and the brain interact to shape who we are. New York, NY: Guilford Press.

Steele, K., Van der Hart, O., \& Nijenhuis, E. R. S. (2001). Dependency in the treatment of complex posttraumatic stress disorder and dissociative disorders. Journal of Trauma and Dissociation, 2(4), 79-116.

Steele, K., Van der Hart, O., \& Nijenhuis, E. R. S. (2005). Phase-oriented treatment of structural dissociation in complex traumatization: Overcoming trauma-related phobias. Journal of Trauma and Dissociation, 6(3), 11-53.

Twombly, J. H. (2000). Incorporating EMDR and EMDR adaptations into the treatment of clients with dissociative 
identity disorder. Journal of Trauma \& Dissociation, 1(2), 61-80.

Twombly, J. H. (2005). EMDR for clients with dissociative identity disorder, DDNOS, and ego states. In R. Shapiro (Ed.), EMDR solutions: Pathways to healing (pp. 88-120). New York, NY: Norton.

Twombly, J. H. (2010). Installation and transmission of current time and life orientation. In M. Luber (Ed.), Eye movement desensitization and reprocessing (EMDR) scripted protocols: Special populations (pp. 243-244). New York, NY: Springer Publishing.

Van der Hart, O., \& Boon, S. (1997). Treatment strategies for complex dissociative disorders: Two Dutch case examples. Dissociation, 10, 157-165.

Van der Hart, O., Groenendijk, M., Gonzalez, A., Mosquera, D., \& Solomon, R. (2013). Dissociation of the personality and EMDR therapy in complex traumarelated disorders: Applications in phase 1 treatment. Journal of EMDR Practice and Research, 7, 81-94.

Van der Hart, O., Nijenhuis, E. R. S., \& Solomon, R. M. (2010). Dissociation of the personality in complex trauma-related disorders and EMDR: Theoretical consideration. Journal of EMDR Practice \& Research, 4, 76-92.

Van der Hart, O., Nijenhuis, E. R. S., \& Steele, K. (2006). The haunted self: Structural dissociation and the treatment of chronic traumatization. New York, NY: Norton.

Van der Hart, O., Steele, K., Boon, S., \& Brown, P. (1993). The treatment of traumatic memories: Synthesis, realization and integration. Dissociation, 6, 162-180.

Van der Kolk, B. A., Spinazzola, J., Blaustein, M. E., Hopper, J. W., Hopper, E. K., Korn, D. L., \& Simpson W. B. (2007). A randomized clinical trial of eye movement desensitization and reprocessing (EMDR), fluoxetine, and pill placebo in the treatment of posttraumatic stress disorder: Treatment effects and long-term maintenance. Journal of Clinical Psychiatry, 68, 37-46.

Young, W. (1994). EMDR treatment of phobic symptoms in multiple personality. Dissociation, 7, 129-133.

Zuschriften zu diesem Artikel bitte an: Onno van der Hart, Department of Clinical and Health Psychology, Utrecht University, Heidelberglaan 1, 3584 CS Utrecht, The Netherlands. E-mail: o.vanderhart@uu.nl 